\title{
Petri Net Supervisors for DES with \\ Uncontrollable and Unobservable Transitions
}

\author{
Technical Report of the ISIS Group \\ at the University of Notre Dame \\ ISIS-99-004 \\ http://www.nd.edu/ isis/tech.html \\ February, 1999
}

John O. Moody

Lockheed Martin Federal Systems

1801 State Rt. 17C, MD 0210

Owego, NY 13827-3998

john.moody@lmco.com
Panos J. Antsaklis

Department of Electrical Engineering

University of Notre Dame

Notre Dame, IN 46556

Panos.J.Antsaklis.1@nd.edu

\section{Interdisciplinary Studies of Intelligent Systems}

The partial financial support of the National Science Foundation (ECS95-31485) and the Army Research Office (DAAG55-98-1-0199) is gratefully acknowledged. 


\title{
Petri Net Supervisors for DES with Uncontrollable and Unobservable Transitions
}

\begin{abstract}
A supervisor synthesis technique for Petri net plants with uncontrollable and unobservable transitions that enforces the conjunction of a set of linear inequalities on the reachable markings of the plant is presented. The approach is based on the concept of Petri net place invariants. Each step of the procedure is illustrated through a running example involving the supervision of a robotic assembly cell. The controller is described by an auxiliary Petri net connected to the plant's transitions, providing a unified Petri net model of the closed loop system. The synthesis technique is based on the concept of admissible constraints. An inadmissible constraint can not be directly enforced on a plant due to the uncontrollability or unobservability of certain plant transitions. Procedures are given for identifying all admissible linear constraints for a plant with uncontrollable and unobservable transitions, as well as methods for transforming inadmissible constraints into admissible ones. When there are multiple transformations of this kind, a technique is described for creating a modified Petri net controller that enforces the union of all these control laws. It is shown how a variety of supervisory control problems can be formulated and solved using the method proposed here. The method is practical and computationally inexpensive in terms of size, design time, and implementation complexity.
\end{abstract}

\section{Introduction}

\section{$1.1 \quad$ Modeling DES with Petri Nets}

It is often necessary to regulate or supervise the behavior of discrete event systems (DES) in order to meet safety or performance criteria, e.g., preventing automated guided vehicles from colliding on a factory floor by restricting their access to certain mutually traveled zones. DES supervisors are used to insure that the behavior of the plant does not violate a set of constraints under a variety of operating conditions. The regulatory actions of the supervisor are based on observations of the plant state, resulting in feedback control.

It is common to see discrete event systems modeled as finite automata [22,28]. Methods exist for designing controllers based on automata system models, however these methods often involve exhaustive searches or simulations of system behavior, making them impractical for systems with large numbers of states and transition causing events.

Modeling discrete event systems with Petri nets (PN's) may help address some of these difficulties. Petri nets $[3,20,21,24]$ have a simple mathematical representation employing linear matrix algebra making them particularly useful for analysis and design. Petri net models are normally more compact than automata based models that represent the same system behavior and are better suited for the representation of systems with repeated structures and flows but large reachable state spaces. Petri nets allow for the simultaneous occurrence of multiple events without suffering from increased model complexity, as is the case with automata. In addition they have an appealing graphical representation that makes it possible to visualize the state-flow of a system and to quickly see dependencies of one part of a system on another. 
The intuitive graphical representation and the powerful algebraic formulation of Petri nets has lead to their use in a number of practical fields. Petri nets are used to model multiprocessor computer systems, computer networks, digital communication protocols, process control plants, queuing systems, and flexible manufacturing cells, among others. Often times the graphical representation of a plant as a Petri net model is enough for an engineer to design a controller or supervisor for the plant. Many control techniques exist that involve recognizing and then manipulating certain structures that commonly appear in Petri net models. Other techniques exist for automatically verifying the reliability of these control designs. A survey of a variety of a variety of supervisory control procedures for Petri nets can be found in [8]. Representing the controller itself as a Petri net makes the verification of the combined plant/controller system simpler, as well as reducing the number of computational tools required to model the overall system. Unfortunately, even when the controller is modeled as a Petri net, this cyclic technique of design and verification can become quite cumbersome when the plant model is large. This leads to the desire for an efficient method for the automatic generation of controllers based on the plant and constraint data.

\subsection{Invariant Based Controllers and Linear State Constraints}

A method for automatically deriving supervisory controllers for discrete event systems described by Petri nets appears in $[19,32]$. The control designer is presented with a Petri net model of a DES and a set of linear constraints on the state space of the DES, and the control goal is to insure that the constraints are met during the plant's operation. The method is based on the idea that specifications representing desired plant behaviors can be enforced by making them place invariants of the feedback controlled system. The resulting controllers are themselves Petri nets and are identical to the monitors [7] of Giua et al., which were derived independently using a different methodology. This technique forms the basics of the synthesis procedure described in this paper and is summarized in section 3 .

Linear inequalities can be used to describe a large class of forbidden state problems. The basic synthesis procedure requires that the set of allowed states be a convex region described by the conjunction of several linear inequalities. The modified control structure of section 6 expands the class of realizable forbidden state problems by allowing nonconvex feasible regions in the form of disjunctions of linear state inequalities. Thus the control procedure can be applied to many common problems seen in flexible manufacturing, process control, and communication networks, including serial, parallel and general mutual exclusion problems $[4,7]$, and the modeling and allocation of shared resources [17].

Insuring system liveness or avoiding deadlock is a common and important supervisory control goal. The existence of liveness-insuring supervisors for Petri nets with uncontrollable transitions has been studied by Sreenivas [26,27]. Techniques for deadlock avoidance have been proposed by a variety of researchers; see $[1,2,5,10,11,29]$ for details. These techniques involve analysis of the siphons or other similar structures within the Petri net plant. Often the resulting controllers can be expressed in terms of supervisors enforcing sets of linear inequalities on the reachable plant states. Combining these techniques with the supervision approach of this paper can then be used to prevent deadlock or insure liveness for plants with uncontrollable and unobservable transitions.

Some supervisory control specifications may seem outside the scope of the technique presented in 
this paper because they are not normally expressed as linear inequalities or because they deal with events or time, rather than particular states. However many of these problems can be expressed as constraints on the state and enforced using the invariant based controllers of this paper. Established and systematic techniques exist for transforming a class of logical predicates on plant behavior into linear state inequalities [31]. Conditions involving the occurrence of events and particular regions of the state space or conditions involving the concurrence of individual events can also be expressed as state-based inequalities [15,32]. The addition of "clock" and "timer" structures to timed Petri nets provides an interface between continuous time and the event driven world of supervisory control. This allows for the synthesis of controllers for timed Petri nets with control specifications involving real time $[15,16]$.

A major goal in the field of discrete event system control is the synthesis of supervisors under conditions where certain state to state transitions can not be prevented by any action from the supervisor, i.e., conditions under which certain transitions are uncontrollable. The problem is then to design a controller that prevents states from occurring that violate the behavioral constraints directly or that might lead to a violation of the constraints through the action of uncontrollable transitions.

Li and Wonham [13] have made important contributions regarding the enforcement of linear constraints on Petri net plants with uncontrollable transitions. These authors show that optimal, or maximally permissive, control actions that account for uncontrollable transitions can be found by repeated applications of an integer linear programming problem (ILP), assuming that valid control actions actually exist and that the uncontrollable portion of the net contains no loops. They also give sufficient conditions under which the solution to the ILP has a closed form expression. In these cases, the control law can be enforced by a feedback Petri net supervisor of the type described in section 3 or 6 of this paper. The computation of the control law, described in sections 4 and 5 presented here involves only matrix algebra and is more desirable, computationally, than analytically solving an ILP. The tree structure assumed by Li and Wonham is only sufficient, not necessary, for example, the structure of the uncontrollable part of the plant in section 6.2 does not have a "tree structure," in fact it contains a loop, however a maximally permissive supervisor was found and implemented using a modified Petri net of the kind described in section 6 .

The concept of uncontrollability is associated with the dual concept of unobservability. It is possible that a DES plant might contain certain state to state transitions that can not be detected by the supervisor. The mathematical representation of these unobservable events is analogous to uncontrollable transitions. Both uncontrollable and unobservable transitions are covered by the design procedures of this paper.

\subsection{Summary of Contents}

Following the review of the algebraic model of Petri nets in section 2, a brief summary of the basic synthesis procedure of [32] appears in section 3. The primary contribution of this paper is the extension of these results to the synthesis of PN supervisors for plants with uncontrollable and unobservable transitions. One possible approach to this problem is to construct a supervisor that searches through the uncontrollably reachable markings of the plant at every iteration of the plant's evolution. This potentially expensive search is avoided here through the concept of 
admissible constraints, introduced in section 4. A constraint is called admissible when, among the states that satisfy the constraint, none could lead (uncontrollably) to a state that does violate the constraint. Admissible constraints may be simply and directly enforced on a plant without requiring that the supervisor search through uncontrollably reachable markings. Computational techniques for generating admissible constraint transformations are presented in the appendix, and supervisors for enforcing admissible constraints can be synthesized using the technique of section 3 .

Section 5 shows how to characterize all admissible linear constraints for a given Petri net. When a constraint is found to be inadmissible, this characterization can be used to find the set of all admissible constraints that have feasible regions that lie within the feasible region of the original constraint. Section 6 shows how to construct a supervisor that will enforce the logical union of all these admissible constraints (a disjunction of linear inequalities), thus providing for a high degree of plant freedom while accounting for uncontrollable and unobservable transitions.

Section 7 shows how a variety of supervisory control problems can be formulated and solved using the proposed method. These problems include the modeling of finite resources, constraints on allowed events (as opposed to states), constraints expressed as logical predicates on plant behavior, and real time constraints for timed Petri nets.

Concluding remarks are given in section 8.

\section{$2 \quad$ Petri Net Fundamentals}

A Petri net is a directed bipartite graph. The structure of a Petri net is described by $\left(P, T, D^{+}, D^{-}\right)$ where $P$ and $T$ are disjoint sets representing the vertices of the graph, known as places and transitions, and $D^{+}$and $D^{-}$are integer matrices with nonnegative elements representing the flow relation between the two vertex types.

Places in a Petri net hold tokens, the distribution of which indicates the net's state or its marking. Transitions direct the flow of tokens between places, thus the firing of a transition is a state changing event in a DES model. A Petri net's incidence matrix represents the weighted connections of directed arcs between its places and transitions. It is composed of two matrices, $D^{-}$, representing arcs from places to transitions, and $D^{+}$, representing arcs from transitions to places.

$$
D=D^{+}-D^{-} \quad\left(D^{+}, D^{-} \geq 0\right)
$$

The incidence matrix is used to construct a difference equation that describes the evolution of the net's state.

$$
\begin{aligned}
\mu(k+1) & =\mu(k)+D q(k) \\
\mu(0) & =\mu_{0}
\end{aligned}
$$

where

$$
\mu \in \mathbb{Z}^{n}, q \in \mathbb{Z}^{m}, \mu, q \geq 0
$$

where $\mathbb{Z}$ is the set of integers, $\mu$ is the net's state or marking vector, $q$ is the input or firing vector,

and $D \in \mathbb{Z}^{n \times m}$. The notation $\mu, q \geq 0$ indicates that every element of the marking and firing 
vectors is nonnegative at all times. This element-by-element interpretation of inequalities will be used throughout this paper whenever vectors or matrices appear on either side of the inequality symbol.

The nonnegativity conditions in (2) lead to the Petri net transition enabling rule. A firing vector $q$ is feasible (represents a valid set of transition firings) if $q \geq 0$ and

$$
D^{-} q \leq \mu
$$

where the iteration counter $k$ has been dropped for convenience. If the Petri net's transitions contain no self loops, i.e., the positions of the nonzero elements in $D^{+}$and $D^{-}$are mutually exclusive, then the transition enabling rule can be written

$$
\mu+D q \geq 0
$$

Care must be taken when using (3) or (4) when $q$ indicates the concurrent firing of multiple transitions. There are a variety of different techniques for handling concurrency. Concurrency may not be allowed at all, in which case $q$ would be a zero vector with a single element equal to one. Concurrency may be allowed only when each of the indicated transition firings could occur one after the other in any order. In this case $q$ must satisfy (3), or each transition firing indicated in $q$ must independently satisfy (4) as well as the complete $q$ vector. If (4) is used without this check for independently enabled transitions, then certain concurrent firings may be allowed even though some or all of the individual transitions indicated in the firing could not fire by themselves. The choice of which of these methods to use is dictated by the modeling requirements and the particular plant.

Petri net place invariants are fundamental to the supervisor synthesis technique described in the following section. A place invariant is an integer vector $x$ that satisfies

$$
\forall \text { reachable } \mu, x^{T} \mu=x^{T} \mu_{0}
$$

Thus $x^{T} \mu$ is a constant for all reachable states if $x$ is a place invariant. Place invariants can be computed by finding solutions to the equation

$$
x^{T} D=0
$$

\section{Invariant Based Control Synthesis}

\subsection{Description of Method}

The system in need of supervision, the plant net, is modeled by a Petri net with $n$ places and $m$ transitions. The plant's incidence matrix is $D_{p} \in \mathbb{Z}^{n \times m}$. The controller net is a Petri net with incidence matrix $D_{c} \in \mathbb{Z}^{n_{c} \times m}$ made up of the plant's transitions and a separate set of places. The controlled system or controlled net is the Petri net with incidence matrix $D \in \mathbb{Z}^{\left(n+n_{c}\right) \times m}$ made up of both the original plant and the added controller. The control goal is to force the plant to obey constraints of the form

$$
L \mu_{p} \leq b
$$


where $\mu_{p} \in \mathbb{Z}^{n}, \mu_{p} \geq 0$ is the marking vector of the plant, $L \in \mathbb{Z}^{n_{c} \times n}$, and $b \in \mathbb{Z}^{n_{c}}$. The inequality is with respect to the individual elements of the two vectors $L \mu_{p}$ and $b$ and can be thought of as the logical conjunction of $n_{c}$ separate linear inequalities.

Inequality (5) can be transformed into an equality by introducing an external Petri net controller that contains places that represent nonnegative "slack variables." The constraint then becomes

$$
L \mu_{p}+\mu_{c}=b
$$

where $\mu_{c} \in \mathbb{Z}^{n_{c}}, \mu_{c} \geq 0$ is the marking of the controller. Note that $\mu_{c} \geq 0$ because the number of tokens in a PN place can not become negative; thus equation (6) implies inequality (5). The closed loop system has the following Petri net structure.

$$
D=\left[\begin{array}{l}
D_{p} \\
D_{c}
\end{array}\right] \quad \mu_{0}=\left[\begin{array}{l}
\mu_{p_{0}} \\
\mu_{c_{0}}
\end{array}\right]
$$

The controller is computed by observing that the introduction of slack variables forces a set of place invariants on the overall controlled system defined by equation (6). The results in the propositions below have been introduced and discussed in $[15,18,19,32]$

\section{Theorem 1. Invariant based controller synthesis. If}

$$
b-L \mu_{p_{0}} \geq 0
$$

then a Petri net controller, $D_{c} \in \mathbb{Z}^{n_{c} \times m}$ with initial marking $\mu_{c_{0}} \in \mathbb{Z}^{n_{c}}$

$$
\begin{gathered}
D_{c}=-L D_{p} \\
\mu_{c_{0}}=b-L \mu_{p_{0}}
\end{gathered}
$$

enforces constraint (5) when included in the closed loop system (7), assuming that the plant's transitions are controllable and observable.

If inequality (8) is not true, then the constraints can not be enforced by any controller since the initial conditions of the plant lie outside the range allowed by the constraints.

Proof. If inequality (8) is not true, then obviously $L \mu_{p_{0}} K b$, and there is at least one row of $L, l_{i}$, such that $l_{i}^{T} \mu_{p_{0}}>b_{i}$ and the initial conditions of the plant violate the constraint. If the inequality is true, then equation (10) shows that the initial conditions of the controller are defined as a vector representing the slack in each of the constraints represented by $L \mu_{p} \leq b$.

Equation (9) forces equation (6) to be place invariants of the closed loop system (see $[18,19,32]$ ), thus inequality (5) will be true for all reachable markings of the closed loop system.

Proposition 2. Invariant based controllers are maximally permissive. Given a plant and a set of enforceable constraints (5), a controller constructed according to the rules of Theorem 1 only acts to disable transitions when the firing indicated by the given $q$ vector leads to a state forbidden by (5). 
Proof. According to the rules of PN evolution, the controller will only disable transitions when the firing indicated by $q$ would cause the marking of at least one of its places to become negative. Since (6) represents a set of invariants in the closed loop system, any negative element in $\mu_{c}$ indicates a violation of (5), and all states allowed by (5) correspond to nonnegative values in $\mu_{c}$.

It has also been shown in [32] that the controller only induces place invariants in the closed loop system that are specifically described by equation (6). All place invariants of the closed loop system are accounted for by those originally present in the plant and those specifically required to enforce the constraints.

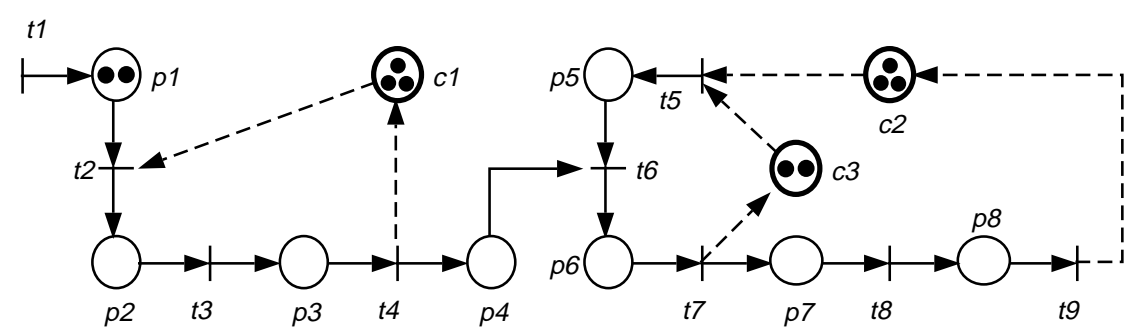

Figure 1: The piston rod robotic assembly cell with its initial controller.

\subsection{Example - The Piston Rod Robotic Assembly Cell}

The piston rod assembly cell application is partially based on a similar plant described in chapter 8 of [4]. The Petri net model of the plant is shown in Figure 1, and Table 1 details the meaning of each place in the net. The number of tokens in each place signifies the number of resources or robots engaged in the activities described in Table 1. The assembly of each part requires work by two different robots. An S-380 robot is used to prepare and align the parts for assembly, and an M-1 robot installs the cap on the piston rod. Places $c_{1}, c_{2}$ and $c_{3}$ in Figure 1 are the components of a supervisory controller, the design of which is covered here.

\begin{tabular}{|l|l|}
\hline$p_{1}$ & Engine block and crank shaft are ready to be processed. \\
\hline$p_{2}$ & S-380 robot aligns the crank shaft. \\
\hline$p_{3}$ & S-380 robot picks up new piston rod and positions it in work space. \\
\hline$p_{4}$ & Engine block prepared and ready for work by M-1 robot. \\
\hline$p_{5}$ & M-1 robot picks up a piston pulling tool. \\
\hline$p_{6}$ & M-1 robot pulls the piston rod into the engine block, returns pulling tool. \\
\hline$p_{7}$ & M-1 robot positions a cap on the piston rod. \\
\hline$p_{8}$ & M-1 robot bolts the cap to the piston rod. \\
\hline
\end{tabular}

Table 1: Place Descriptions for Figure 1.

There are three S-380 and three M-1 robots available in the assembly cell. There are two piston pulling tools. These resource constraints are translated into linear inequalities on the state space 
of the plant:

$$
\begin{array}{rlrl}
\mu_{2}+\mu_{3} & \leq 3 & & \text { (three S-380 robots) } \\
\mu_{5}+\mu_{6}+\mu_{7}+\mu_{8} \leq 3 & & \text { (three M-1 robots) } \\
\mu_{5}+\mu_{6} \leq 2 & & \text { (two piston pulling tools) }
\end{array}
$$

Each inequality is enforced by a separate controller place. The connections of these three places, $c_{1}, c_{2}$, and $c_{3}$, to the plant and their initial markings are calculated using Theorem 1 , resulting in the maximally permissive supervisor shown in Figure 1.

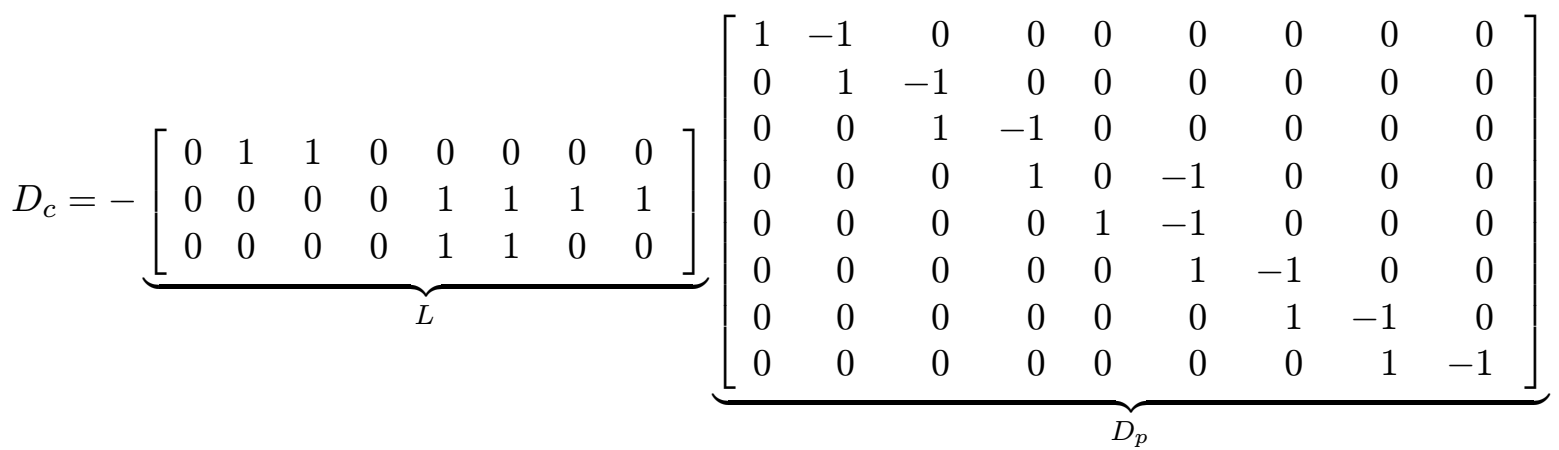

$$
\begin{aligned}
& =\left[\begin{array}{rrrrrrrrr}
0 & -1 & 0 & 1 & 0 & 0 & 0 & 0 & 0 \\
0 & 0 & 0 & 0 & -1 & 0 & 0 & 0 & 1 \\
0 & 0 & 0 & 0 & -1 & 0 & 1 & 0 & 0
\end{array}\right] \\
& \mu_{c 0}=\underbrace{\left[\begin{array}{l}
3 \\
3 \\
2
\end{array}\right]}_{b}-L \mu_{p 0}=\left[\begin{array}{l}
3 \\
3 \\
2
\end{array}\right]
\end{aligned}
$$

\section{Admissible Constraints and Controls}

\subsection{Uncontrollable and Unobservable Transitions}

A transition is called uncontrollable if the firing of that transition may not be inhibited by an external action. The freedom of an uncontrollable transition to fire is limited solely by the structure and state of the plant.

In order for a Petri net controller to inhibit a transition, it must contain an arc from a controller place to the transition. The transition will be disabled if the number of tokens in the control place is less than the arc weight.

A transition is called unobservable if the firings of that transition can not be directly detected or measured. Since the firing of an unobservable transition can not be detected, a controller state change can not be triggered by such a firing.

For a Petri net based controller, both input and output arcs to the plant transitions are used to trigger state changes in the controller. A Petri net controller can not have any connections to 
an unobservable transition, thus all unobservable transitions are also implicitly uncontrollable, of course an uncontrollable transition may or may not be unobservable. One can imagine a situation where the occurrence of some event in a plant could be blocked without the controller ever receiving any feedback relating directly to that event, but, in practical situations, the ability to inhibit an event is usually coupled with the ability to detect occurrences of that event. For this reason, this limitation on Petri net based controllers is not too severe.

\subsection{Constraint Transformations}

Given a set of constraints, $L \mu_{p} \leq b$, a supervisor must work to insure that the constraints are never violated directly and may never be violated through the firing of uncontrollable transitions or through incomplete knowledge due to unobservable transitions. In order to avoid expensive online searches by the supervisor through the uncontrollably reachable markings of the plant, the approach taken here is to actually modify the constraints themselves such that the new constraints account for uncontrollability and unobservability. The following definitions are useful in understanding the motivation for the transformation of constraints. The definitions are with respect to a plant with possible uncontrollable or unobservable transitions and constraints on the marking behavior of the plant in the form $L \mu_{p} \leq b$. Unobservable transitions are also assumed to be uncontrollable.

Definition 3. An admissible marking $\mu_{p}$ is a marking such that

1. $L \mu_{p} \leq b$, and

2. For all markings $\mu_{p}^{\prime}$ reachable from $\mu_{p}$ through the firing of uncontrollable transitions, $L \mu_{p}^{\prime} \leq b$.

If either of these conditions is not met, then the marking is inadmissible.

Definition 4. Given a plant with initial marking $\mu_{p}(0)=\mu_{p_{0}}$, an admissible constraint satisfies two conditions:

1. $L \mu_{p_{0}} \leq b$, and

2. For all $\mu_{p}(N)$ reachable from $\mu_{p}(0)$ through any path of consecutively reachable markings, $\mu_{p}(0) \rightarrow \mu_{p}(1) \rightarrow \cdots \rightarrow \mu_{p}(N)$, where

$$
L \mu_{p}(i) \leq b, \text { for } 1 \leq i \leq N
$$

$\mu_{p}(N)$ is an admissible marking.

If a constraint does not satisfy both of these conditions, then it is inadmissible.

If a constraint is admissible, then condition 2 of Definition 4 indicates that the firing of uncontrollable transitions can never lead from a state that satisfies the constraint to a new state 
that violates that constraint. Note that the admissibility of a particular marking does not imply that the marking is actually reachable, either due to the initial marking of the plant or due to the restrictions of a supervisor.

An admissible constraint will only allow admissible markings, however there may exist admissible markings that could be reached by the uncontrolled plant that can not be reached under maximally permissive supervision. Definition 4 incorporates this by checking the admissibility of markings that were achieved by following paths in which all intermediate markings satisfy the constraint. This set of reachable, admissible markings is similar to the set $\operatorname{Re}(G, P)$ defined in [12].

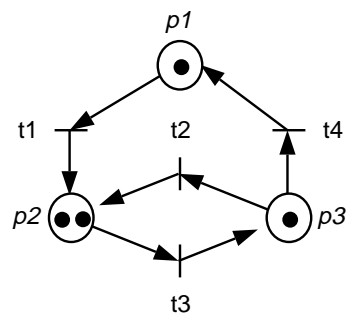

Figure 2: Transitions 2 and 3 are uncontrollable.

Example. The Petri net of Figure 2 contains two uncontrollable transitions: $t_{2}$ and $t_{3}$. Tokens in places $p_{2}$ and $p_{3}$ can not be prevented from freely traveling between these two places. However $t_{1}$ can be used to stop the introduction of new tokens into $p_{2}$ and $p_{3}$, and $t_{4}$ can be used to prevent tokens from leaving.

The constraint

$$
\mu_{3} \leq 1
$$

is inadmissible. The initial state of the plant $\mu_{0}=\left[\begin{array}{lll}1 & 2 & 1\end{array}\right]^{T}$ satisfies the constraint, but the uncontrollable firing of $t_{3}$ would lead to the state $\mu=\left[\begin{array}{lll}1 & 1 & 2\end{array}\right]^{T}$, which violates (14). The constraint fails condition 2 of Definition 4.

The constraint

$$
\mu_{1} \leq 1
$$

is admissible. The current state of the plant satisfies the constraint, and for any state that satisfies the constraint, there is no firing of uncontrollable transitions that would lead to a state that does not satisfy it. The marking of $p_{1}$ is affected only by the firings of transitions $t_{1}$ and $t_{4}$, both of which are controllable.

If $L \mu_{p} \leq b$ is inadmissible, then it is desirable to find another constraint $L^{\prime} \mu_{p} \leq b^{\prime}$ such that $L^{\prime} \mu_{p} \leq b^{\prime}$ is an admissible constraint, and for all $\mu_{p}$ such that $L^{\prime} \mu_{p} \leq b^{\prime}, L \mu_{p} \leq b$ is also true. In the example above, we could replace constraint (14) with

$$
\mu_{2}+\mu_{3} \leq 1
$$


This constraint is admissible according to Definition 4, and all reachable states that satisfy (16) also satisfy (14). Thus constraint (14) could be enforced by designing a controller for constraint (16) using the technique of section 3. Unfortunately a controller designed this way may not be maximally permissive. The method of handling uncontrollable/unobservable transitions in section 5 of this paper follows along these lines, but it also includes the idea of finding all constraints $L^{\prime} \mu_{p} \leq b^{\prime}$ that meet the criteria above. Section 6 then shows how to construct a controller that enforces the disjunction of these inequalities allowing for a high degree of plant freedom.

\subsection{Petri Net Modeled Supervisors}

The supervisors used in this paper are modeled by Petri nets. Uncontrollable and unobservable transitions can cause problems for PN based supervisors due to limitations in their modeling power, however Petri net supervisors are still useful for several reasons. Unified plant/controller models are elegant, facilitating implementation and closed loop system analysis. The evolution of Petri net models is inexpensive to compute, facilitating their use in real time control applications. Desirable Petri net qualities, such as automatic handling of concurrent events, are maintained with unified plant/controller PN models. Though the decision power of a Petri net supervisor is not unlimited, a good variety of DES control problems can be effectively and efficiently solved through their use. Recognizing the controller as a Petri net facilitates understanding of what can and can not be done with the supervisor. This will become evident in the material below.

For an invariant based Petri net supervisor to be realizable on a plant with uncontrollable and unobservable transitions, the constraint it is enforcing must be admissible. Proposition 5 provides necessary and sufficient conditions for any behavioral constraint to be admissible. Definitions 3 and 4 are written specifically for linear state-based constraints, however, they can be thought of in terms of general behavioral constraints. That is, Definition 3 requires that a particular marking and all behaviors achieved through the firing of uncontrollable transitions from that marking conform to the constraint. Definition 4 requires that the initial condition of a plant satisfy the constraint, and that all markings visited through any behavior that conforms to the constraint are admissible markings.

The behavior of a maximally permissive supervisor is analyzed in Proposition 5. Here the term maximally permissive is used in the sense of section 3, where all transitions are assumed to be controllable. In this case, a maximally permissive controller only prevents firings that lead to states that directly violate the given constraint.

Proposition 5. General constraint admissibility. A constraint on the marking and/or firing behavior of a Petri net is admissible iff

1. The initial conditions of the plant satisfy the constraint, and

2. There exists a maximally permissive controller (constructed under the assumption that all transitions are controllable) that enforces the constraint and does not inhibit any uncontrollable transitions that would otherwise be enabled. 
Proof. Clearly, if the initial conditions of a plant violate a constraint, then that constraint can not be enforced and is inadmissible according to condition 1 of Definition 4. Furthermore, if the constraint is admissible, then a maximally permissive controller would have no need to attempt to disable otherwise enabled uncontrollable transitions, as per Definition 4.

A maximally permissive controller will only allow reachable states or behaviors that do not violate the constraint. Thus, if a maximally permissive controller never attempts to inhibit an otherwise enabled uncontrollable transition, then the constraint it is enforcing is admissible according to Definition 4.

Corollary 6. Place-constraint admissibility. The single vector constraint $l^{T} \mu_{p} \leq b$ is $a d$ missible iff the controller with incidence matrix $D_{c}=-l^{T} D_{p}$ and initial marking $\mu_{c 0}=b-l^{T} \mu_{p 0} \geq 0$ will never attempt to disable an uncontrollable transition that would otherwise be enabled.

Proof. If $\mu_{c 0} \geq 0$, then the initial conditions of the plant violate the constraint, and that constraint can not be enforced and is inadmissible according to condition 1 of Definition 4 . Invariant based controllers are maximally permissive according to Proposition 2; if the constraint is admissible, then this maximally permissive controller would have no need to attempt to disable otherwise enabled uncontrollable transitions, as per Definition 4.

Invariant based controllers only allow reachable states that do not violate the constraint by inhibiting the firing of any transition that would directly lead to a marking that violates the constraint. Thus, if it never attempts to inhibit an otherwise enabled uncontrollable transition, then the constraint it is enforcing is admissible according to Definition 4.

Remark. Corollary 6 deals with individual inequality constraints rather than the vector inequality $L \mu_{p} \leq b$ because each of the inequalities in $L \mu_{p} \leq b$ can be handled independently. Certain constraints in $L \mu_{p} \leq b$ may be admissible, while others may not.

Equations (9) and (10) from Theorem 1 show that it is possible to construct the incidence matrix $D_{c}$ of a maximally permissive Petri net controller as a linear combination of the rows of the incidence matrix of the plant. Negative elements in $D_{c}$ correspond to arcs from controller places to plant transitions. These arcs act to inhibit plant transitions when the corresponding controller places are empty, and thus they can only be applied to plant transitions that permit such external control. If we group all of the columns of $D_{p}$ that correspond to transitions that can not be controlled into the matrix $D_{\mathrm{uc}}$, we obtain the following corollary.

Corollary 7. $\quad l^{T} D_{\mathrm{uc}} \leq 0$ implies admissibility. Given a plant with uncontrollable transitions described by the incidence matrix $D_{\mathrm{uc}}$ and a constraint $l^{T} \mu_{p} \leq b$, if

$$
l^{T} D_{\mathrm{uc}} \leq 0
$$

then the constraint is admissible for the given plant. 
Proof. The proof follows from Corollary 6 and the construction of the Petri net controller whose incidence matrix is $D_{c}=-l^{T} D_{p}$ as described in section 4.1. Inequality (17) insures that the controller draws no arcs to uncontrollable transitions.

Example. Corollary 7 can be used to verify the results from the example in section 4.2. Since transitions $t_{2}$ and $t_{3}$ are uncontrollable in the Petri net of Figure 2, $D_{\mathrm{uc}}$ is composed of the second and third columns of the plant incidence matrix.

$$
D_{p}=\left[\begin{array}{rrrr}
-1 & 0 & 0 & 1 \\
1 & 1 & -1 & 0 \\
0 & \underbrace{-1}_{D_{\mathrm{uc}}} & 1
\end{array}\right]
$$

Constraint (14) fails to meet condition (17) of the corollary.

$$
\left[\begin{array}{lll}
0 & 0 & 1
\end{array}\right] D_{\mathrm{uc}}=\left[\begin{array}{ll}
-1 & 1
\end{array}\right]
$$

Constraints (15) and (16) both meet condition (17) and are both admissible.

$$
\begin{aligned}
& {\left[\begin{array}{lll}
1 & 0 & 0
\end{array}\right] D_{\mathrm{uc}}=\left[\begin{array}{ll}
0 & 0
\end{array}\right]} \\
& {\left[\begin{array}{lll}
0 & 1 & 1
\end{array}\right] D_{\mathrm{uc}}=\left[\begin{array}{ll}
0 & 0
\end{array}\right]}
\end{aligned}
$$

Remark. Corollary 7 provides only a sufficient condition for constraint admissibility. There exist situations for which $l^{T} D_{\mathrm{uc}} \not \leq 0$, but $l^{T} \mu_{p} \leq b$ is still an admissible constraint (see $[15,16]$ ). However, for most practical examples, constraints that fail condition (17) are inadmissible and will need to be transformed if they are to be enforced.

As discussed in section 4.1, it is illegal for the controller to change its state based on the firing of an unobservable transition, because there is no direct way for the controller to be told that such a transition has fired. Both input and output arcs from the controller places are used to change the controller state based on the firings of plant transitions. Let the matrix $D_{\text {uo }}$ represent the incidence matrix of the unobservable portion of the Petri net. This matrix is composed of the columns of $D_{p}$ that correspond to unobservable transitions, just as $D_{\mathrm{uc}}$ is composed of the uncontrollable columns of $D_{p}$.

Corollary 8. $\quad l^{T} D_{\mathrm{uo}}=0$ implies admissibility. Given a plant with unobservable transitions described by the incidence matrix $D_{\mathrm{uo}}$ and a constraint $l^{T} \mu_{p} \leq b$, if

$$
l^{T} D_{\mathrm{uo}}=0
$$

then the constraint is admissible. 
Proof. As with Corollary 7, the proof follows from Corollary 6 and the construction of the Petri net controller whose incidence matrix is $D_{c}=-l^{T} D_{p}$ as described in section 4.1. Equation (18) insures that the controller draws no arcs to or from unobservable transitions.

Remark. Corollaries 7 and 8 indicate that it is possible to observe a transition that we can not inhibit, but it is illegal to directly inhibit a transition that we can not observe.

Suppose, given a set of constraints $L \mu_{p} \leq b$, we construct the matrices $L D_{\mathrm{uc}}$ and $L D_{\mathrm{uo}}$ and observe that there are violations to conditions (17) and/or (18). What other constraints, of the form $L^{\prime} \mu_{p} \leq b^{\prime}$, will also maintain the original constraint $L \mu_{p} \leq b$ ?

\section{Lemma 9. Constraint transformation structure.}

Let $R_{1} \in \mathbb{Z}^{n_{c} \times n}$ satisfy $R_{1} \mu_{p} \geq 0 \forall \mu_{p}$.

Let $R_{2} \in \mathbb{Z}^{n_{c} \times n_{c}}$ positive definite diagonal matrix

If $L^{\prime} \mu_{p} \leq b^{\prime}$ where

$$
\begin{aligned}
L^{\prime} & =R_{1}+R_{2} L \\
b^{\prime} & =R_{2}(b+\mathbf{1})-\mathbf{1}
\end{aligned}
$$

and 1 is an $n_{c}$ dimensional vector of 1's, then $L \mu_{p} \leq b$.

Proof. The transformed constraint is $\left(R_{1}+R_{2} L\right) \mu_{p} \leq R_{2}(b+\mathbf{1})-\mathbf{1}$. Because all of the elements are integers, the inequality can be transformed into a strict inequality:

$$
\left(R_{1}+R_{2} L\right) \mu_{p}<R_{2}(b+\mathbf{1})
$$

Because $R_{2}$ is diagonal and positive definite,

$$
R_{2}^{-1} R_{1} \mu_{p}+L \mu_{p}<b+\mathbf{1}
$$

Assumptions (19) and (20) imply that all elements of the vector $R_{2}^{-1} R_{1} \mu_{p} \geq 0$, therefore $L \mu_{p} \leq b$.

Lemma 9 shows a class of constraints, $L^{\prime} \mu_{p} \leq b^{\prime}$, which, if enforced, will imply that $L \mu_{p} \leq b$ is also enforced. The following lemma is used to show the conditions under which a particular set of constraints can be enforced on a particular Petri net.

Lemma 10. Initial condition check for transformed constraints. The constraint $L^{\prime} \mu_{p} \leq$ $b^{\prime}$, where $L^{\prime} \neq 0$ and $b^{\prime}$ are defined by (21) and (22), can be enforced on a Petri net with initial marking $\mu_{p_{0}}$ iff

$$
0 \leq R_{1} \mu_{p_{0}} \leq R_{2}\left(b+\mathbf{1}-L \mu_{p_{0}}\right)-\mathbf{1}
$$


Proof. Substituting $L^{\prime}$ and $b^{\prime}$ into (23) gives $0 \leq b^{\prime}-L^{\prime} \mu_{p_{0}}$, which is equivalent to the condition $L^{\prime} \mu_{p_{0}} \leq b^{\prime}$ that states that the initial conditions of the plant must fall within the acceptable region of the constraints. Clearly, if a controller does exist, then the initial conditions of the plant must not violate the constraints. Furthermore, as shown in section 3, if the initial conditions lie within the acceptable region of the plant (inequality (8)), a controller to enforce the conditions can be computed with incidence matrix $D_{c}=-L^{\prime} D_{p}$ and initial marking $\mu_{c_{0}}=b^{\prime}-L^{\prime} \mu_{p_{0}}$.

Theorem 11 combines Corollaries 7 and 8 with the conditions for creating a valid set of transformed constraints in lemmas 9 and 10 to show how to construct a Petri net controller.

Theorem 11. Constraint transformation and supervisor synthesis. Let a plant Petri net with incidence matrix $D_{p}$ be given with a set of uncontrollable transitions described by $D_{\mathrm{uc}}$ and a set of unobservable transitions described by $D_{\text {uo }}$. A set of linear constraints on the net marking, $L \mu_{p} \leq b$, are to be imposed. Assume $R_{1}$ and $R_{2}$ meet (19) and (20) with $R_{1}+R_{2} L \neq 0$ and let

$$
\left[\begin{array}{ll}
R_{1} & R_{2}
\end{array}\right]\left[\begin{array}{cccc}
D_{\mathrm{uc}} & D_{\mathrm{uo}} & -D_{\mathrm{uo}} & \mu_{p_{0}} \\
L D_{\mathrm{uc}} & L D_{\mathrm{uo}} & -L D_{\mathrm{uo}} & L \mu_{p_{0}}-b-\mathbf{1}
\end{array}\right] \leq\left[\begin{array}{cccc}
0 & 0 & 0 & \mathbf{- 1}
\end{array}\right]
$$

Then the controller

$$
\begin{gathered}
D_{c}=-\left(R_{1}+R_{2} L\right) D_{p}=-L^{\prime} D_{p} \\
\mu_{c_{0}}=R_{2}(b+\mathbf{1})-\mathbf{1}-\left(R_{1}+R_{2} L\right) \mu_{p_{0}}=b^{\prime}-L^{\prime} \mu_{p_{0}}
\end{gathered}
$$

exists and causes all subsequent markings of the closed loop system (7) to satisfy the constraint $L \mu_{p} \leq b$ without attempting to inhibit uncontrollable transitions and without detecting unobservable transitions.

Proof. According to (9) and (10), equations (25) and (26) define a controller that enforces the constraint $L^{\prime} \mu_{p} \leq b^{\prime}$. Lemma 9 shows that if assumptions (19) and (20) are met then a controller that enforces a particular constraint $L^{\prime} \mu_{p} \leq b^{\prime}$ will also enforce the constraint $L \mu_{p} \leq b$. The fourth column of inequality (24) indicates that the condition in lemma 10 is satisfied, thus the controller exists and the control law can be enforced. The first column of (24) indicates that $L^{\prime} D_{\mathrm{uc}} \leq 0$, thus condition (17) is satisfied and no controller arcs are drawn to the uncontrollable transitions. The second and third columns of (24) indicate that $L^{\prime} D_{\mathrm{uo}}=0$, thus condition (18) is satisfied and no arcs are drawn between the controller places and the unobservable plant transitions.

Remark. [ $\left.\begin{array}{ll}R_{1} & R_{2}\end{array}\right]$, which is used to describe the constraint transformation, is a left multiplier in (24), thus this matrix represents the use of rows from $D_{\mathrm{uc}}$ to eliminate positive elements from $L D_{\mathrm{uc}}$, and the use of rows from $D_{\mathrm{uo}}$ to zero the elements of $L D_{\mathrm{uo}}$.

The usefulness of Theorem 11 for specifying controllers to handle plants with uncontrollable and unobservable transitions lies in the ease in which the matrices $R_{1}$ and $R_{2}$ can be generated. Two computational techniques for computing these matrices can be found in the appendix. The first technique is an integer program that searches through feasible solutions satisfying (17) and 
(18) along directions dictated by (23). The second technique finds appropriate transformations through the use of constrained integer row operations. Full details of each algorithm are included in the appendix.

\subsection{Example - Uncontrollable and Unobservable Transitions in the Assembly Cell}

Uncontrollable and unobservable transitions are introduced into the robotic assembly cell example from section 3.2. The operation of the M-1 robots is now considered to be governed by a separate, independent controller. Transitions $t_{6}, t_{7}$, and $t_{8}$ can neither be observed nor inhibited by the resource supervisor of section 3.2.

The uncontrollable and unobservable portion of the plant is described by the matrix $D_{\mathrm{uo}}$, which is composed of the sixth through eighth columns of $D_{p}$. Of the three constraints, (11), (12), and (13), only (13) fails the test of Corollary 8 , since

$$
\left[\begin{array}{llllllll}
0 & 0 & 0 & 0 & 1 & 1 & 0 & 0
\end{array}\right] D_{\mathrm{uo}}=\left[\begin{array}{lll}
0 & -1 & 0
\end{array}\right]
$$

If the plant transitions were merely uncontrollable and not unobservable as well, then the constraint would be admissible according to Corollary 7 , but Corollary 8 indicates that $l^{T} D_{\text {uo }}=0$ is the sufficient condition for admissibility with unobservable transitions.

The right hand side of (27) can be zeroed by adding to it the seventh and eighth rows of $D_{\mathrm{uc}}$. In terms of Theorem 11, this corresponds to a constraint transformation using

$$
R_{1}=\left[\begin{array}{llllllll}
0 & 0 & 0 & 0 & 0 & 0 & 1 & 1
\end{array}\right] \quad R_{2}=1
$$

The transformed constraint,

$$
\mu_{5}+\mu_{6}+\mu_{7}+\mu_{8} \leq 2
$$

is admissible and represents the maximally permissive admissible control law for enforcing (12). The new configuration for $c_{3}$ is shown in Figure 3. Note that places $c_{2}$ and $c_{3}$ now have the exact same connections to the plant. It would be possible to eliminate $c_{2}$, since its action is now implied by $c_{3}$, but instead, both places will be maintained in order to account for dynamic changes in the number of available resources or sensors (see $[16,17])$.

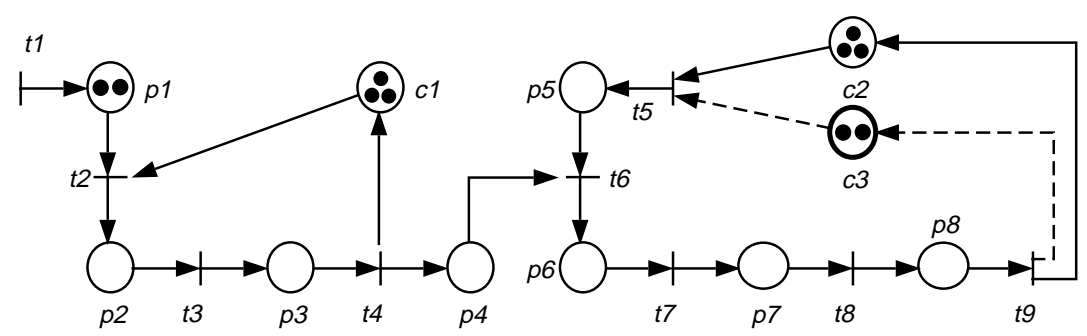

Figure 3: The modified assembly cell and supervisor after the introductions of uncontrollable and unobservable transitions. 


\section{Structure of Admissible Constraints and Controls}

Given a plant with uncontrollable/unobservable transitions, it is useful to seek methods for transforming inadmissible constraints into admissible ones, but it is also logical to ask, in general, what are the admissible constraints for this plant? Is there a way to characterize all or most of these constraints? Section 5.1 provides a method for just such a characterization. Section 5.2 shows how this characterization may be used to synthesize controllers for plants with unobservable transitions, and section 5.3 covers the synthesis problem for plants with uncontrollable transitions.

\subsection{Characterization of Admissible Constraints}

As in the previous sections, let the matrix $D_{\text {uo }}$ represent the incidence matrix of the unobservable portion of the Petri net. It is illegal for the controller $D_{c}=-L D_{p}$ to contain any arcs in the unobservable portion of the net, thus an admissible set of constraints will satisfy

$$
L D_{\mathrm{uo}}=0
$$

as indicated in Corollary 8.

Any $L$ that satisfies (29) will lie within the kernel of $D_{\text {uo }}$. Let $X$ satisfy

$$
X D_{\mathrm{uo}}=0
$$

where $X \in \mathbb{Z}^{\left(n-\text { rank } D_{\text {uo }}\right) \times n}$. The rows of $X$ form a linearly independent basis for the kernel of $D_{\text {uo }}$ ( $X$ is full rank). The process of finding $X$ is equivalent to finding the place invariants (an algorithm appears in [14]) of the unobservable portion of the plant Petri net. All realizable constraints must lie within the basis described by the rows of $X$, and thus can be formed as linear combinations of these rows. Every admissible constraint can be described by $k^{T} X$ where $k \in \mathbb{Z}^{\left(n-\text { rank } D_{\text {uo }}\right)}$. In general, the coefficient matrix of any set of admissible constraints $L^{\prime} \in \mathbb{Z}^{n_{c} \times n}$ can be written

$$
L^{\prime}=K X
$$

where $K \in \mathbb{Z}^{n_{c} \times\left(n-\operatorname{rank} D_{\text {uo }}\right)}$.

A characterization of all admissible constraints is not quite as transparent for the case of uncontrollable transitions as it is for unobservable ones. For unobservable transitions we have an equality, $L D_{\text {uo }}=0$, which must be satisfied, but for uncontrollable transitions it is an inequality, $L D_{\mathrm{uc}} \leq 0$, so we can not simply find the kernel of $D_{\mathrm{uc}}$. In this case, the following equality can be formed

$$
L D_{\mathrm{uc}}+\Delta=0
$$

where $\Delta$ is a matrix of nonnegative slack variables. The previous equation is rewritten

$$
\left[\begin{array}{ll}
L & \Delta
\end{array}\right]\left[\begin{array}{c}
D_{\mathrm{uc}} \\
I
\end{array}\right]=0
$$

A kernel $X$, solving

$$
X\left[\begin{array}{c}
D_{\mathrm{uc}} \\
I
\end{array}\right]=0
$$


can then be used to construct a basis for all admissible linear constraints that may be placed on the plant. $X$ must be computed so as to insure that the elements that correspond to $\Delta$ are nonnegative. Each element of the kernel will have $n+n_{\mathrm{uc}}$ scalar components, where $n_{\mathrm{uc}}$ is the number of uncontrollable transitions. The final $n_{\mathrm{uc}}$ elements of each kernel vector correspond to the slack variables in $\Delta$. Additional row operations may need to be performed on $X$ to insure that the final $n_{\mathrm{uc}}$ elements in each vector are nonnegative. After insuring that none of the slack variables are negative, all admissible constraint matrices $L$ can be found in the the linear combinations of $X$ that leave nonnegative values in the final $n_{\mathrm{uc}}$ slack columns. The first $n$ components of a given kernel vector represent an admissible value for a row of $L$, as long as the final $n_{\mathrm{uc}}$ components of the kernel vector are nonnegative.

\subsection{Constraint Transformations for Unobservability}

Suppose we have a set of constraints $L \mu \leq b$ such that $L D_{\text {uo }} \neq 0$. It is necessary to create new constraint matrices $\left(L^{\prime}, b^{\prime}\right)$ with two properties.

1. $L^{\prime} D_{\mathrm{uo}}=0$

2. $\forall \mu_{p}, L^{\prime} \mu_{p} \leq b^{\prime} \rightarrow L \mu_{p} \leq b$

Property 1 is necessary to insure that the new controller will not utilize the unobservable transitions, while property 2 indicates that the new constraints must be at least as restrictive as the original ones. Lemma 9 from section 4.3 is used to deal with this condition.

To perform the transformation, it is necessary to determine values for the matrices $R_{1}$ and $R_{2}$ defined in lemma 9 that meet assumptions (19) and (20). It is possible for a designer to determine the values of $R_{1}$ and $R_{2}$ by using the kernel of $D_{\text {uo }}$. Combining equations (21) and (31) we see that

$$
L^{\prime}=K X=R_{1}+R_{2} L
$$

The designer should premultiply each constraint in $L$ by some positive integer (which will determine the diagonal elements in $R_{2}$ ) and add new positive coefficients (which will determine $R_{1}$ ) such that the new constraint is a linear combination of the rows of $X$. This process will yield the $L^{\prime}$ matrix, and $b^{\prime}$ can be calculated using $R_{2}$ and equation (22).

When multiple distinct transformations exist, the technique of section 6 can be used to enforce the disjunction of all these inequalities.

\subsection{Constraint Transformations for Uncontrollability}

The invariant based control method yields maximally permissive supervisors for enforcing linear constraints of the form $L \mu_{p} \leq b$. When these constraints are transformed, because of the uncontrollability and unobservability of certain transitions, into $L^{\prime} \mu_{p} \leq b^{\prime}$, the invariant based control method will still yield a maximally permissive realization of the transformed constraint. Unfortunately, the new constraint itself may not represent the most permissive admissible control law 
corresponding to the original constraint. The maximally permissive admissible constraint associated with a linear predicate on the plant's marking may be a nonlinear predicate that can not be optimally controlled by a standard invariant based controller.

At this time, a complete description of the conditions under which an optimal transformation of linear constraints is another set of linear constraints is unknown. Li and Wonham [13] have shown that when the uncontrollable portion of the plant has a "type 1 tree structure," the optimal transformation will be a disjunction of linear predicates ${ }^{1}$, while a "type 2 " structure will yield a linear transformation. However these conditions are only sufficient, not necessary.

Given an inadmissible constraint $l^{T} \mu_{p} \leq b$, a permissive control law for the enforcement of this constraint can be synthesized using the following steps.

1. Find all inequalities $l^{\prime T} \mu_{p} \leq b^{\prime}$ that are

(a) Valid transformations of $l^{T} \mu \leq b$ according to Lemma 9 and

(b) Admissible constraints according to the theory developed in section 5.1.

There may be an infinite number of inequalities that meet these two requirements, but they may be expressed with a finite number of inequalities since linearly dependent constraints do not represent different restrictions on the behavior of the plant. Detailed instructions for carrying out this step can be found in [16].

2. Construct the controller incidence matrices associated with these constraints using $D_{c}=$ $-l^{\prime T} D_{p}$.

3. Enforce the union of the individual control laws by following the procedure of section 6 .

The procedure above is similar to the idea of the supremal controllable sublanguage $[22,28]$ from the supervisory control literature. In both cases, all of the valid behaviors of the plant are characterized based on the plant's structure and the desired constrained behavior, and the supervisor is then used to insure that the behavior of the plant is limited to this set of admissible behaviors.

To say that the procedure above will always result in a maximally permissive control law, the following two points would have to be proved.

1. The maximally permissive control law associated with a plant and constraint $l^{T} \mu_{p} \leq b$ can always be expressed as the disjunction of other linear state inequalities.

2. The transformation procedure in Lemma 9 covers all valid constraint transformations, i.e., if for all $\mu_{p} \geq 0$ such that $l^{\prime T} \mu_{p} \leq b^{\prime}, l \mu_{p} \leq b$ is also true, then $\left(l^{\prime}, b^{\prime}\right)$ can be expressed as a linear function of $(l, b)$ according to the rules and assumptions of Lemma 9.

Li and Wonham [13] have shown that condition 1 is true when the uncontrollable portions of a plant have a certain "tree structure" (see [13]). But for the general case, the answer is not known.

\footnotetext{
${ }^{1} \mathrm{~A}$ Procedure for enforcing these with a modified PN controller is presented in section 6 .
} 


\section{Enforcing Disjunctions of Linear Constraints}

\subsection{Description of Method}

The inequality

$$
L \mu_{p} \leq b
$$

represents the logical intersection, or conjunction, of $n_{c}$ separate linear inequalities. That is, if $l_{i}$ is the $i^{\text {th }}$ row of $L$ and $b_{i}$ is the $i^{\text {th }}$ element of $b$, then (32) is equivalent to

$$
\bigwedge_{i=1}^{n_{c}} l_{i}^{T} \mu_{p} \leq b_{i}
$$

The feasible solutions to the inequalities form a convex region [6], and the behavior of a Petri net can be restricted to this region by adding further $\mathrm{PN}$ structures to the net as was shown in section 3. A logical union, or disjunction, of linear constraints is, in general, nonconvex and can not be enforced with maximal permissivity on a Petri net through the use of other Petri net structures due to the linear nature of reachable PN state spaces. A proof of this claim appears in [15].

This section will show how a slight modification to the evolution rules of the controller net can be made such that it will act as a maximally permissive supervisor for a class of nonconvex constraints.

The following disjunction of linear inequalities is to be enforced on the marking of a plant with initial marking $\mu_{p_{0}} \in \mathbb{Z}^{n}$ (all elements nonnegative) and incidence matrix $D_{p} \in \mathbb{Z}^{n \times m}$.

$$
\bigvee_{i=1}^{n_{c}} l_{i}^{T} \mu_{p} \leq b_{i}
$$

where $l_{i} \in \mathbb{Z}^{n}$ and $b_{i} \in \mathbb{Z}$. Let

$$
D_{c_{i}}=-l_{i}^{T} D_{p}
$$

and

$$
\mu_{c_{i 0}}=b_{i}-l_{i}^{T} \mu_{p_{0}}
$$

for $1 \leq i \leq n_{c}$. This procedure is the same as detailed in section 3 , thus each pair $\left(D_{c_{i}}, \mu_{c_{i}}\right)$ is a maximally permissive PN supervisor for enforcing the constraint $l_{i}^{T} \mu_{p} \leq b_{i}$. However if all of these supervisor elements were to be simultaneously enforced on the plant, then the result would be the logical intersection of the constraints rather than their union.

In order for the controller to enforce a disjunction of inequalities, at least one of the inequalities $l_{i}^{T} \mu_{p} \leq b_{i}$ must be true at every transition firing iteration of the net's evolution. Let

$$
L=\left[\begin{array}{llll}
l_{1} & l_{2} & \cdots & l_{n_{c}}
\end{array}\right]^{T}
$$

so that

$$
D_{c}=-L D_{p}
$$

and

$$
\mu_{c_{0}}=b-L \mu_{p_{0}}
$$


which is identical to the controller construction from section 3. However, the enabling rule for the controller portion of the net must be changed such that it insures that at least one of the inequalities is being obeyed at all times rather than all of them at all times.

A firing vector $q$ is valid (indicates the firing of an enabled transition) iff

$$
D_{p}^{-} q \leq \mu_{p}
$$

and

$$
\mu_{c_{i}}+D_{c_{i}} q \geq 0 \text { for some } i \in 1 \ldots n_{c}
$$

Inequality (38) is the standard PN enabling condition for a plant that may include transitions with self loops. The enabling condition for the controller (39) does not include any $D_{c_{i}}^{-}$terms because controllers constructed according to the rules in section 3 do not contain self loops. Condition (39) may also be written

$$
\max _{i=1}^{n_{c}}\left(\mu_{c_{i}}+D_{c_{i}} q\right) \geq 0
$$

Note that it is still true that

$$
L \mu_{p}+\mu_{c}=b
$$

however, unlike the standard nonnegative slack variables from before, many of the elements of this $\mu_{c}$ may be negative. The restriction placed by condition (40) insures that at least one of the elements is nonnegative, and thus at any time, at least one of the inequalities in (33) is being satisfied.

Proposition 12. Maximal permissivity of disjunction-enforcing controllers. A controller constructed according to (36) and (37) using enabling rule (40) is a maximally permissive supervisor for the enforcement of constraint (33) on the plant $\left(D_{p}, \mu_{c_{0}}\right)$ iff

$$
\mu_{c_{i_{0}}} \geq 0 \text { for some } i \in 1 \ldots n_{c} .
$$

Proof. If condition (42) is not met, then the initial conditions of the plant violate the constraint (33) according to equation (37), and the constraint can not be enforced.

Equation (41) shows that the state space of the closed loop system being outside the bounds of constraint (33) is equivalent to the situation when all the elements of $\mu_{c}$ are negative. However this is the only condition that is prevented by enabling rule (40). The only time the controller will intervene to disable a transition is when the firing of that transition would cause a direct violation of constraint (33), and thus the supervisor is maximally permissive.

Remark. The simple rules that govern ordinary Petri net behavior are what help to make the PN model so attractive both for analysis and implementation. The reluctance to modify this model for the enforcement of nonconvex constraints on PN plants is overcome for the following reasons.

1. The ability to handle the disjunction of linear constraints as well as their conjunction is a powerful advancement in the utility of the method and is necessary for the proper solution of problems in many applications. 
2. Disjunctions of linear constraints are important for the permissive enforcement of linear constraints under conditions in which transitions may be uncontrollable or unobservable.

3. The modified rules for controller state evolution involve only a slight modification of the ordinary transition enabling rule. Analysis and implementation are very similar to that of ordinary Petri nets.

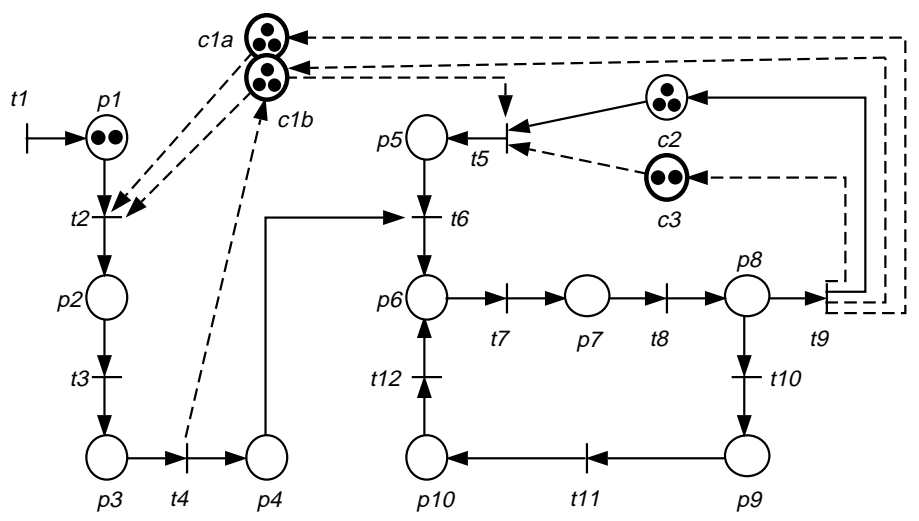

Figure 4: The final structure of the assembly cell and supervisor. Places $c_{1 a}$ and $c_{1 b}$ obey the modified PN enabling rule (40) in order to enforce the nonconvex constraint (45).

\subsection{Example - An Uncontrollable Loop is Added to the Assembly Cell}

The robots of the piston rod robotic assembly cell are not $100 \%$ reliable. It is possible that the M-1 robot will fail to properly secure a piston cap to its rod. The plant is now augmented with an error recovery loop that is considered to be under the supervision of an auxiliary controller. The modified structure is shown in Figure 4. The uncontrollable firing of transition $t_{10}$ indicates that a fault has occurred. Place $p_{9}$ is then marked with the number of M-1 robots that have experienced faults and have entered the recovery loop. Tokens in $p_{10}$ represent the combined actions of M-1 and S-380 robots to replace and realign the appropriate parts so that the procedure can begin again at $p_{6}$. The new transitions, $t_{10}, t_{11}$, and $t_{12}$ are all considered uncontrollable and unobservable to the resource managing supervisor.

Constraints (11) and (12) need to be rewritten to account for the use of the two robots in the recovery loop. An S-380 robot is used in $p_{10}$, and the M-1 robot is required in both $p_{9}$ and $p_{10}$. The new constraints are then

$$
\begin{aligned}
\mu_{2}+\mu_{3}+\mu_{10} & \leq 3 & & \text { (three } \mathrm{S}-380 \text { robots }) \\
\mu_{5}+\mu_{6}+\mu_{7}+\mu_{8}+\mu_{9}+\mu_{10} & \leq 3 & & \text { (three } \mathrm{M}-1 \text { robots })
\end{aligned}
$$

Following the procedure of section 5.2, the kernel, $X$, of the unobservable incidence matrix, 
$D_{\text {uo }}$, is computed:

$$
\underbrace{\left[\begin{array}{llllllllll}
1 & 0 & 0 & 0 & 0 & 0 & 0 & 0 & 0 & 0 \\
0 & 1 & 0 & 0 & 0 & 0 & 0 & 0 & 0 & 0 \\
0 & 0 & 1 & 0 & 0 & 0 & 0 & 0 & 0 & 0 \\
0 & 0 & 0 & 1 & 0 & 1 & 1 & 1 & 1 & 1 \\
0 & 0 & 0 & 0 & 1 & 1 & 1 & 1 & 1 & 1
\end{array}\right]}_{X} D_{\text {uo }}=0
$$

Admissible constraints will be linearly dependent with the rows of $X$. The left hand side of (44) is equal to row five of $X$, thus the constraint is admissible and requires no transformation. In order to make the left hand side of (43) an element of the kernel of $D_{\mathrm{uo}}$, we can either add the missing elements from row four or row five of $X$. Because there is a choice, the transformed constraint will be written as a disjunction of the two candidate inequalities.

$$
\left(\mu_{2}+\mu_{3}+\mu_{4}+\mu_{6}+\mu_{7}+\mu_{8}+\mu_{9}+\mu_{10} \leq 3\right) \vee\left(\mu_{2}+\mu_{3}+\mu_{5}+\mu_{6}+\mu_{7}+\mu_{8}+\mu_{9}+\mu_{10} \leq 3\right)
$$

A controller is calculated to enforce (45) using the procedure described in section 6.1. The supervisor, shown with its connections to the plant in Figure 4, is maximally permissive.

\section{Control Specifications}

Constraints of the form $L \mu_{p} \leq b$ (inequality (5)) are useful for representing a large variety of forbidden state problems. This section will show how several common varieties of system constraints can be written in the form of (5), enabling the use of invariant based control.

Section 7.1 shows how the management of finite resources can be handled using invariant based control. Section 7.2 discusses equality constraints and demonstrates how attempts to enforce them with invariant based controllers can lead to deadlock. Section 7.3 explains how both direct and indirect enforcement of constraints on events, i.e., linear inequalities involving the firing vector, can be handled using the invariant based control method. In section 7.4, a class of logical predicates on plant behavior are transformed into systems of linear inequalities to be enforced by a supervisor. Section 7.5 explains how the techniques for supervision of ordinary Petri nets can be expanded to timed Petri nets and real-time constraints.

\subsection{Modeling of Finite Resources}

A finite resource is a tool or material with limited supply that is required by one or more agents for the completion of a job or to carry out some action. The availability of finite resources places implicit constraints on feasible actions within a system. These constraints can be written as linear inequalities on the state. Let $b_{i}$ be the total number of available units for resource $i$. Let $R_{i}$ be a set of places associated with finite resource $i$. Every token in the places making up the set $R_{i}$ represents the use of one of the resources. A linear constraint on the marking can then be written

$$
\sum_{\mu_{j} \in R_{i}} \mu_{j} \leq b_{i}
$$


Suppose that a resource suddenly becomes available or the number of available resources changes in some other way during the operation of the plant. This situation could be handled by modifying the token count in the appropriate controller slack place, i.e., the number $b_{i}$ on the right hand side of (46) could be modified dynamically. According to Theorem 1, dynamic modifications to $b$ in a constraint inequality will not change the structure of the controller. The arcs and their associated weights will remain the same. The only change would be in the marking of a controller place to correspond to the new slack value. Though this scheme would work, it is not very elegant from the point of view of Petri nets, i.e., tokens should not appear and disappear from a net without the corresponding firing of transitions.

Figure 5 shows how the resource controller places can be augmented with two uncontrollable transitions and a place in order to model the loss of finite resources while maintaining the standard Petri net framework of the model. Under normal operation, the token in the resource place is used to permit the firing of the plant transition. However the loss of the finite resource now corresponds to the firing of an uncontrollable transition which robs the "resource is available" place of its token and stalls the operation of the plant. Another uncontrollable transition is then used to replace the missing token when the resource once again becomes available.

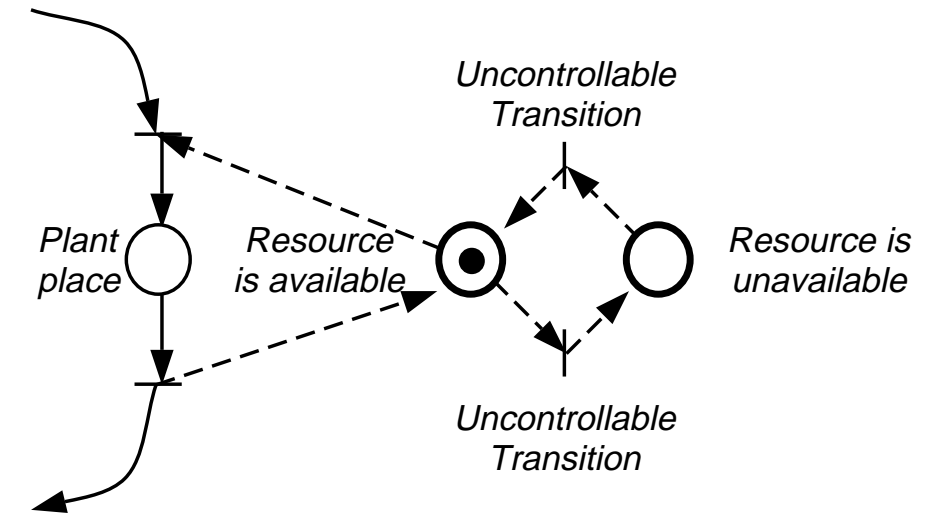

Figure 5: Modeling the loss of a finite resource using uncontrollable transitions.

Remark. It may seem odd that the structure shown in Figure 5 contains uncontrollable transitions connected directly to the PN controller places. The reason these transitions have been marked uncontrollable is because it may not be possible to know when they will fire, and it does not matter to the controller. The "Resource is available" place in the controller represents the standard invariant based controller place that keeps track of the slack associated with its given constraint. The "Resource is unavailable" place can "steal" tokens from the primary place, thus reducing the slack and reducing the number of resources that can be allocated to the plant. The associated transitions here may be considered uncontrollable, but they still must obey standard PN rules, thus a resource will not suddenly become unavailable if it is currently in use in the plant. If resources that are currently in use may become unavailable at any time, the modeling and management of them must be handled differently by incorporating this behavior in the plant model itself. 


\subsection{Equality Constraints}

Though rare, in some circumstances the control designer may wish to insure that the token count in a set of places remains constant. For example, suppose we have a Petri net describing the task distribution in a multiprocessor computer, and the designer wants to insure that there are always two processors (no more and no less) available to handle I/O. Consider a chemical processing plant where tokens representing reactants in a set of places must be kept constant to maintain a desired chemical equilibrium. Constraints like these may take the form of an equalities, rather than inequalities.

Equality constraints have the form

$$
L \mu_{p}=b
$$

Equation (47) defines place invariants on the original process net. This is really a specification for the system and should have been incorporated into the Petri net model before attempting to use supervisory control. If this invariant is not already part of the Petri net model, it should become one by modifying the incidence matrix $D_{p}$ of the plant. The new elements of $D_{p}$ represent the arcs that should be added to the Petri net so that the place invariants are enforced.

It may seem feasible to use the place invariant control method to force $L \mu_{p} \leq b$ and $L \mu_{p} \geq b$ to achieve the constraint of equation (47). Unfortunately this approach will produce undesirable results as described by the following proposition.

Proposition 13. Enforcement of equality constraints leads to deadlock. Enforcing constraint (47) by creating invariant based controllers for the constraints

$$
\begin{aligned}
L \mu_{p} & \leq b, \text { and } \\
-L \mu_{p} & \leq-b
\end{aligned}
$$

will

1. have no effect on the plant's behavior, or

2. create a local deadlock in the plant (the system will not be live).

Proof. Suppose that the natural behavior of the plant already meets the desired constraint. In this case, $L$ describes a set of place invariants in the plant and $L D_{p}=0$. Equation (9) shows that the controller for constraint (48) or (49) is given by $D_{c}= \pm L D_{p}=0$. Thus the controller will have no arcs to the plant transitions, and it will have no effect on the plant's behavior.

Now suppose that $L$ does not include natural invariants of the plant. In this case, the controller incidence matrices for (47) and (48) are given by

$$
\begin{aligned}
& D_{c 1}=-L D_{p} \neq 0 \\
& D_{c 2}=L D_{p}=-D_{c 1}
\end{aligned}
$$


Since $D_{c 1}=-D_{c 2}$, all output arcs of the places in $D_{c 1}$ are input arcs of the places in $D_{c 2}$ and vice versa. Thus the set of control places forms a siphon.

The initial marking, $\mu_{p 0}$, of the plant must satisfy $L \mu_{p 0}=b$ or it would not have been feasible to attempt to enforce (47). Equation (10) gives the initial markings of the control places:

$$
\begin{aligned}
& \mu_{c 1_{0}}=b-L \mu_{p 0}=0 \\
& \mu_{c 2_{0}}=-b+L \mu_{p 0}=0
\end{aligned}
$$

Thus the set of control places forms an unmarked siphon and all of the transitions to which these places are connected will be dead.

\subsection{Constraints involving the Firing Vector}

Certain control goals may involve the firing vector of the Petri net as well as or opposed to the places. For example one might need to insure that two transitions do not fire simultaneously or that a certain transition is never allowed to fire when a certain place holds a token. There are two ways that constraints like these may be viewed. For the constraint

$$
\mu_{i}+q_{j} \leq 1
$$

do we mean that transition $j$ should be disabled whenever place $i$ contains a token, or do we mean that all plant states that would allow transition $j$ to be enabled are forbidden whenever place $i$ contains a token? The answer to this question lies in the particulars of a given plant and its operation. Both means of enforcing the constraint can be useful for different problems.

Section 7.3.1 describes rules for enforcing firing vector constraints using the "direct" interpretation, i.e., transitions are explicitly disabled in order to satisfy the inequality. Algebraic schemes for handling the "indirect" interpretation of firing vector constraints were proposed in [32]. A new approach is presented in section 7.3.2 that uses the concept of uncontrollable transitions to force a correct interpretation of each constraint, thus avoiding the enumeration of separate cases that appeared in [32].

\subsubsection{Direct Realization}

Assume that the plant must satisfy constraint (50). The direct interpretation of this constraint implies that transition $t_{j}$ cannot fire if place $p_{i}$ is marked, and, of course, place $i$ can never contain more than one token. To bring this constraint to a form that contains elements of the marking vector only, the plant is transformed as follows. Transition $j$ is replaced by two transitions and a place between them, as shown in Figure 6. This transformation is artificial and will not affect the Petri net model of the process. Its sole purpose is to introduce the place $p_{j}{ }^{\prime}$, which records the firing of the transition $t_{j}$. After the controller has been computed the plant will be transformed back to its original form.

The marking $\mu_{j}{ }^{\prime}$ of $p_{j}{ }^{\prime}$ replaces $q_{j}$ in constraint (50), which becomes

$$
\mu_{i}+\mu_{j}{ }^{\prime} \leq 1
$$




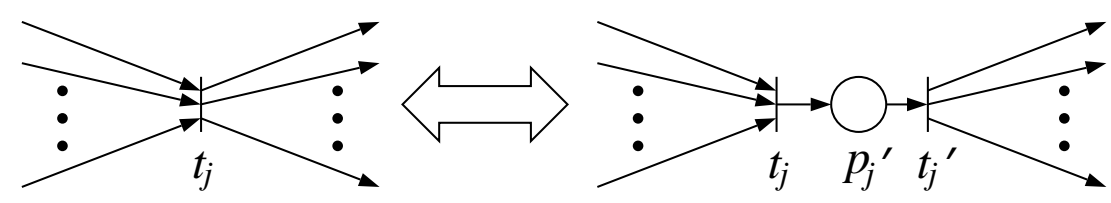

Figure 6: Transformation of a Transition.

The constraint now contains only $\mu$ 's and a controller can now be computed. After the controller structure is computed, the two transitions and the place of the transformation collapse to the original transition thus restoring the original form of the plant while maintaining the enforcement of the new constraint. The same transformation is done to all the transitions that appear in the constraints. Constraints that contain only $q$ 's, i.e., constraints on allowable firing vectors with no concern for specific markings, are treated in the same way.

In terms of Figure 6, output arcs from the controller would normally be connected to transition $t_{j}$, and input arcs to the controller would be connected to $t_{j}^{\prime}$. The act of collapsing the transformed structure back to its original form will cause both the input and output arcs to be connected to the original transition $t_{j}$. Unlike standard invariant based controllers, this means that the controller may contain self loops to the transitions indicated in the constraints. Separate $D_{c}^{+}$and $D_{c}^{-}$matrices must be maintained for the controller and used when determining enabled and disabled transitions.

In summary, given a plant $\left(D_{p}, \mu_{p 0}\right)$ and constraint

$$
l^{T} \mu_{p}+f^{T} q \leq b, f \geq 0
$$

the invariant based controller $\left(D_{c}=D_{c}^{+}-D_{c}^{-}, \mu_{c 0}\right)$ is given by

$$
\begin{aligned}
D_{c}^{+} & =\max \left(0, D_{l c}^{+}-D_{f c}^{-}\right)+\max \left(0, D_{f c}^{+}-D_{l c}^{-}\right) \\
D_{c}^{-} & =\max \left(0, D_{l c}^{-}-D_{f c}^{+}\right)+\max \left(0, D_{f c}^{-}-D_{l c}^{+}\right) \\
\mu_{c 0} & =b-l^{T} \mu_{p 0}
\end{aligned}
$$

where

$$
D_{f c}^{+}=D_{f c}^{-}=f^{T}
$$

and

$$
\begin{aligned}
D_{l c}^{+} & =\max \left(0, D_{l c}\right) \\
D_{l c}^{-}(j) & =\max \left(0,-D_{l c}\right)
\end{aligned}
$$

where

$$
D_{l c}=-l^{T} D_{p}
$$

and the notation $\max (0, a)$ refers to a vector equal to $a$ but with all negative elements replaced with zeros. The equations above account for both the transformation of transitions and the collapse of the transitions back to the original form of the net. Equations (53) and (54) allow arcs from the $D_{l c}$ and $D_{f c}$ portions of the control to cancel each other, but do not allow the self loops in $D_{f c}^{+}$and $D_{f c}^{-}$to cancel each other. 
The remainder of this subsection provides an analysis of the admissibility of firing vector constraints using the direct interpretation. Similar to Corollary 6 , the following corollary defines when a constraint on the firing vector of a Petri net is admissible.

Corollary 14. Transition-constraint admissibility. The single vector constraint $f^{T} q \leq b$, where $f, b \geq 0$, is admissible under direct transition-constraint implementation on a plant with controllable transitions $T_{\mathrm{c}}$, if $\forall j$ s.t. $f_{j} \neq 0, t_{j} \in T_{\mathrm{c}}$.

Proof. The proof is by Proposition 5 on general constraint controllability. The direct transitionconstraint enforcement method for the constraint $f^{T} q \leq b$ is maximally permissive since it is constructed as an invariant based controller. The initial marking of the controller $\mu_{c 0}=b$ is valid if $b \geq 0$. The incidence matrix of the controller $D_{c}^{+}=D_{c}^{-}=f^{T}$ contains input arcs to all transitions $j$ such that $f_{j} \neq 0$. If all of these transitions are controllable, then the controller draws no arcs to uncontrollable transitions and the constraint is admissible.

The admissibility of combined marking/firing constraints, $l^{T} \mu_{p}+f^{T} q \leq b$, will be discussed for the situation in which the constraints are uncoupled.

Definition 15. A constraint of the form (52), where $f \geq 0$, is called uncoupled if

$$
T_{l} \cap T_{f}=\emptyset
$$

where $T_{l}$ is the set of transitions that are connected to the controller induced by the $l^{T} \mu_{p}$ portion of the constraint (transitions $t_{j}$ such that $D_{l c}(j) \neq 0$ in equation (59)), and $T_{f}$ is the set of transitions connected to the controller induced by the $f^{T} q$ portion of the constraint (transitions $t_{j}$ such that $\left.f_{j} \neq 0\right)$.

Constraint (52) is uncoupled if the transitions involved in the $l^{T} \mu_{p}$ and $f^{T} q$ portions of the constraint are mutually exclusive.

Remark. When constraints are uncoupled, equations (53) and (54) reduce to

$$
\begin{aligned}
& D_{c}^{+}=D_{l c}^{+}+D_{f c}^{+} \\
& D_{c}^{-}=D_{l c}^{-}+D_{f c}^{-}
\end{aligned}
$$

Proposition 16. Uncoupled place/transition constraints. A vector constraint of form (52) is uncoupled iff

$$
\forall i \text { s.t. } f_{i} \neq 0, l^{T} D_{p} e_{i}=0
$$

where $e_{i}$ is a zero-vector with a 1 in the $i^{\text {th }}$ place. 
Proof. The set of plant transitions that will contain arcs to or from the controller is determined from the controller synthesis equations. This set is the union of the transitions connected by arcs induced by the $l^{T} \mu_{p}$ and $f^{T} q$ portions of the constraint, i.e., $T_{f} \cup T_{l}$. Equation (56) indicates that

$$
T_{f}=\left\{t_{j} \mid f_{j} \neq 0\right\}
$$

and equations (57) and (58) show

$$
T_{l}=\left\{t_{j} \mid l^{T} D_{p} e_{j} \neq 0\right\}
$$

Combining these with condition (60) implies

$$
T_{l} \cap T_{f}=\emptyset
$$

The sets of transitions used by the two portions of the controller are mutually exclusive and the constraint is uncoupled according to definition 15.

It is easy to see that if the constraints are uncoupled, i.e. $T_{l} \cap T_{f}=\emptyset$, then (60) must be true by working backward through the development above. If (60) were not true, then there would exist some $t_{i} \in T_{f}$ and $t_{i} \in T_{l}$, which would imply through equations (61) and (62) that $T_{l} \cap T_{f} \neq \emptyset$ and the constraints were coupled.

Proposition 17. Place/transition constraint admissibility. An uncoupled vector constraint of form (52) is to be imposed on a plant $\left(D_{p}, \mu_{p 0}\right)$ with uncontrollable transitions $T_{\mathrm{uc}}$ and controllable transitions $T_{\mathrm{c}}, T_{\mathrm{uc}} \cap T_{\mathrm{c}}=\emptyset$.

if the constraints

$$
\begin{aligned}
l^{T} \mu_{p} & \leq b \\
f^{T} q & \leq|b|
\end{aligned}
$$

are both admissible then $l^{T} \mu_{p}+f^{T} q \leq b$ is admissible.

Proof. If the admissibility of constraints (64) and (65) imply that (52) is admissible, then the inadmissibility of (52) will imply that either (64) or (65) is inadmissible or both. For $l^{T} \mu_{p}+f^{T} q \leq b$ to be inadmissible, it must lie outside the range of the plant's initial conditions, or a maximally permissive controller that enforces the constraint would attempt to inhibit an otherwise enabled transition in the set $T_{\mathrm{uc}}$. Because (52) is uncoupled, the transitions that are connected to the controller places, $T_{f}$ and $T_{l}$, are mutually exclusive. This means that at least one of the following three cases must be true for $l^{T} \mu_{p}+f^{T} q \leq b$ to be inadmissible.

1. The initial conditions of the plant violate the constraint.

2. The controller would attempt to inhibit a transition $t_{j} \in T_{\mathrm{uc}}$, where $t_{j} \in T_{f}$.

3. Or the controller would attempt to inhibit an otherwise enabled transition $t_{j} \in T_{\mathrm{uc}}$, where $t_{j} \in T_{l}$. 
Case 1: The initial state of the plant is $\mu_{p 0}$. The firings indicated by the vector $q$ are determined after the system commences its run, thus if the initial conditions of the plant violate constraint (52), then

$$
l^{T} \mu_{p 0}>b
$$

This condition would also indicate that the constraint $l^{T} \mu_{p} \leq b$ is inadmissible according to Corollary 6.

Case 2: According to the construction of the maximally permissive controller for direct transition constraints, the transitions in the set $T_{f}$ are identical to the transitions that receive controller arcs in the constraint $f^{T} q \leq|b|$. If the controller attempts to inhibit an uncontrollable transition in this set, then the constraint $f^{T} q \leq|b|$ is inadmissible according to Corollary 14 .

Case 3: The construction of the maximally permissive controller for the constraint $l^{T} \mu_{p} \leq b$ shows that the transitions that receive controller arcs for this constraint are identical to the set $T_{l}$. If the controller for constraint (52) attempts to disable an otherwise enabled transition in the set $T_{l}$, then the constraint $l^{T} \mu_{p} \leq b$ will be inadmissible according to Corollary 6 .

Thus if both $l^{T} \mu_{p} \leq b$ and $f^{T} q \leq|b|$ are admissible, then $l^{T} \mu_{p}+f^{T} q \leq b$ is also admissible.

The use of the propositions and definitions above are illustrated using a process control example in $[16]$.

\subsubsection{Indirect Realization}

Firing vector constraints can be realized by preventing the states that would allow the undesirable transition firing; this situation is analogous to the case when a transition is uncontrollable but is involved with regular marking constraints. Illegal states are prevented in the presence of uncontrollable transitions by preventing those states which could lead, through uncontrollable transitions, to the explicitly forbidden states. The results for uncontrollable transitions can be applied to constraints involving the firing vector through utilization of the graph transformations discussed in the previous section.

The procedure is illustrated in the example below.

Example. For the plant of Figure 7a, we wish to enforce the constraint

$$
\mu_{2}+q_{3} \leq 1
$$

Place $p_{2}$ is never to have more than one token and transition $t_{3}$ should never fire when this place is occupied. This problem could be solved simply by applying the technique of the previous section, but suppose instead of directly controlling the transition we want to prevent the states that could lead to the constraint being violated. Because the Petri net is so simple, we can see by inspection that the job can be done by enforcing the constraint $\mu_{2}+\mu_{3} \leq 1$. But how can this new constraint be generated automatically based on (66)? 
a)

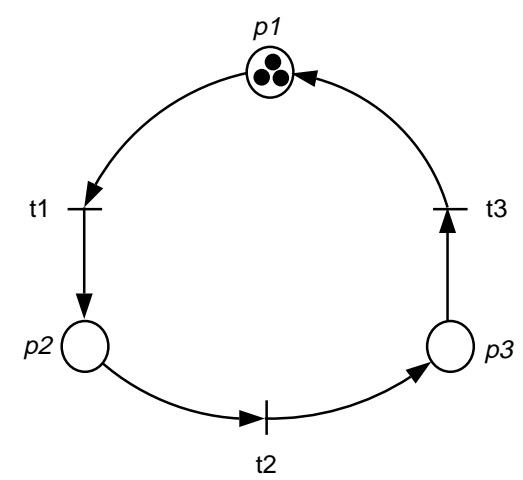

b)

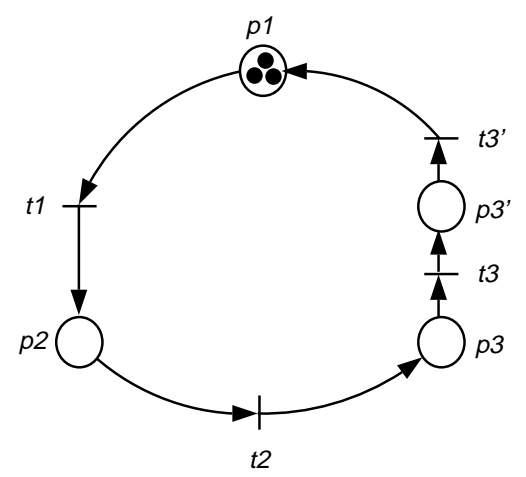

Figure 7: a) A simple net that will have a firing constraint enforced. b) The graph-transformed net of the net in Figure 7a.

Suppose we perform the graph transformation on this net as shown in Figure 7b. The transformation changes $(66)$ to

$$
\mu_{2}+\mu_{3}^{\prime} \leq 1
$$

If we continue to follow the procedure described in section 7.3.1, we would end up with a controller that directly enables and disables transition $t_{3}$. In order to prevent this from occurring, we will label transition $t_{3}$ as uncontrollable and then continue with the procedure.

Applying the method from section 4.3 to (67), we obtain the following transformed constraint:

$$
\mu_{2}+\mu_{3}+\mu_{3}^{\prime} \leq 1
$$

The controller that enforces this constraint can be automatically generated using the place invariant method and is shown in Figure 8a.

The final stage is then to collapse the controlled net back to the form it had before the graph transformation was performed. The final controlled version of the net is shown in Figure $8 \mathrm{~b}$. Transition $t_{3}$ will not fire when place $p_{2}$ contains a token because the controller only allows one token at a time in places $p_{2}$ and $p_{3}$, which is the desired result.

The procedure used in the example is summarized below. Given a constraint

$$
l^{T} \mu_{p}+f^{T} q \leq b
$$

(where $l$ may be zero, indicating a constraint on the firing vector alone,) first perform a transformation of the plant such that each transition specified by a nonzero entry in $f$ includes a dummy place to mark its firing as described in section 7.3.1. The marking vector $\mu^{\prime}$ is associated with the dummy places and the constraint becomes

$$
\left[\begin{array}{ll}
l^{T} & f^{T}
\end{array}\right]\left[\begin{array}{l}
\mu_{p} \\
\mu^{\prime}
\end{array}\right] \leq b
$$


a)

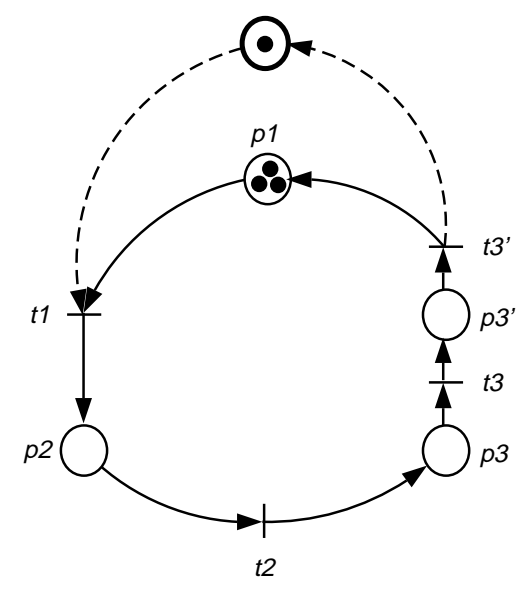

b)

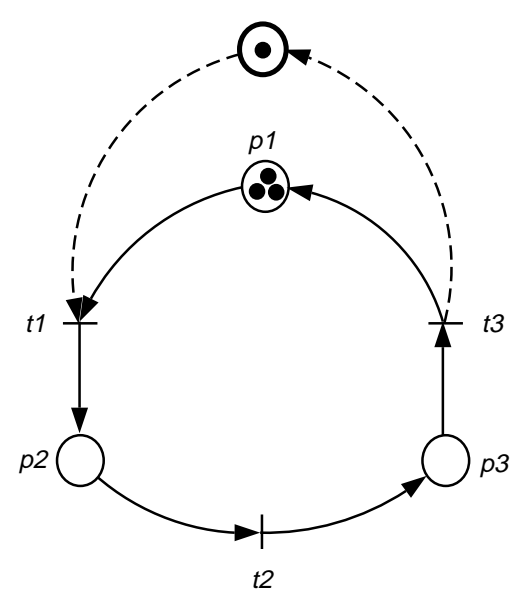

Figure 8: a) The net of Figure 7b with its Petri net controller. b) The untransformed net of Figure 7a with its Petri net controller.

Next mark all transitions specified by nonzero entries in $f$ as uncontrollable. Use established techniques for the handling of uncontrollable transitions to find an admissible constraint that enforces the inadmissible constraint (70) and construct a supervising controller for this constraint. This will have the effect of preventing the states that could lead to (70) being violated. It will prevent the transitions specified by $f$ from being enabled such that constraint (69) could be violated. Finally, collapse the net back to its original form by removing the dummy places and extra transitions as described in section 7.3.1.

\subsection{Logical Constraints on System Behavior}

The transformation of logic-based constraints on system behavior into systems of linear inequalities has been studied by Yamalidou and Kantor [30,31]. These transformations apply to safe nets, meaning that no place in the network can have more than one token at any time. In this case, all places have two states: either they contain a token or they do not. Similarly all transitions can be viewed as having two states: either they will fire in the current iteration of the system's evolution or they will not. This means that both places and transitions have binary valued states in a safe net and they can be viewed as boolean variables.

Consider the simple network in Figure 9a. We wish to enforce the constraint

$$
\text { if } \mu_{1} \neq 0 \text {, then } q_{3}=0
$$

One method of doing this would be to introduce an inhibitor arc into the Petri net model as shown in Figure $9 \mathrm{~b}$. The arc between $p_{1}$ and $t_{3}$ is terminated with a circle indicating that the arc will inhibit the firing of transition 3 whenever place 1 contains any tokens. Unfortunately, with the addition of an inhibitor arc, we are no longer dealing with an ordinary Petri net and have lost some of the ability to analyze the net in a convenient manner due to the increased complexity of the transition enabling rule. 
c)

a)

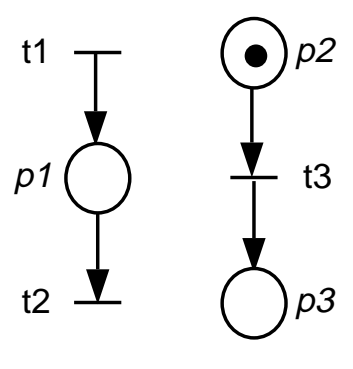

b)

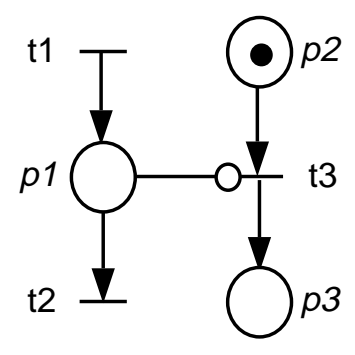

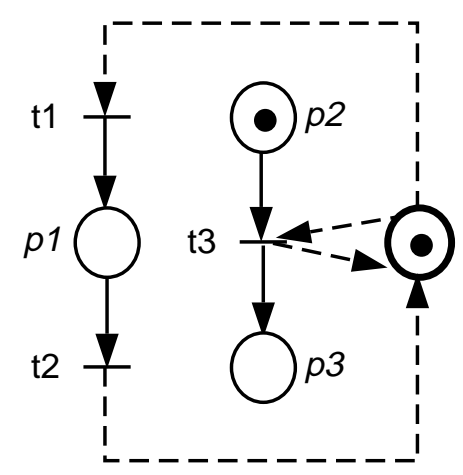

Figure 9: Two methods (b and c) of enforcing constraint (71) on the network in a.

If we assume that the net is safe, constraint (71) can be implemented by transforming it into a linear inequality:

$$
\mu_{1}+q_{3} \leq 1
$$

The controller for enforcing this linear inequality is shown in Figure 9c. It was constructed using the direct method for implementation of firing vector constraints (section 7.3.1).

If the network in Figure 9a were not safe, i.e., transition 1 could fire multiple times allowing tokens to pile up in $p_{1}$, then it is still true that the controller in Figure 9c would enforce constraint (71). Unfortunately it also has the secondary effect of preventing $p_{1}$ from ever containing more than one token. This is due to the literal interpretation of inequality (72) and thus, if the net were not safe, constraint (72) would be more restrictive than constraint (71) and the supervisor could not be considered maximally permissive.

A formal procedure for translating logical implications into linear inequalities appropriate for use as constraints on Petri net behavior appears in [30] (see also [9]). The general form for an implication is

$$
A \rightarrow \Phi
$$

where $A$ is an action (transition firing) or the result of an action (place marking). $\Phi$ is a "well formed" boolean expression describing the state of the system. It contains boolean variables (place markings) and any of the logical operators And, Or, Not, Implication, or Equivalence. Assuming the net is safe, (73) may be transformed into a set of linear inequalities through the following procedure.

First $\Phi$ must be written in conjunctive normal form (product-of-sums form). For example, the boolean expression $A+B C$ would be transformed to $(A+B)(A+C)$. At this point, implication (73) has become

$$
A \rightarrow \phi_{1} \phi_{2} \cdots \phi_{g}
$$


where

$$
\phi_{i}=\psi_{1}+\psi_{2}+\cdots+\psi_{h}
$$

Each variable $\psi$ is boolean and is valued true or false according to the marking of a corresponding Petri net place (which can only be 1 or 0 since the net is assumed to be safe).

The implication can now be broken down into a system of $g$ simultaneous linear inequalities, where $g$ is the number of product terms in (74). Each inequality will have the form

$$
\left(1-\mu_{\psi_{1}}\right)+\left(1-\mu_{\psi_{2}}\right)+\cdots+\left(1-\mu_{\psi_{h}}\right)+A \leq h
$$

where $h$ is number of summed terms in the corresponding $\phi$ value and $\mu_{\psi_{i}}$ is the marking of the place associated with $\psi_{i}$.

Example. A system of inequalities will be derived to enforce the constraint

if transition 1 fires then places 1 and 2 must contain tokens

on a safe Petri net. The action, $A$, is the firing of transition 1 , while $\Phi$ is the logical AND of the truth of places 1 and 2 containing tokens. Though it is a slight notational abuse, for convenience the implication is expressed as

$$
q_{1} \rightarrow \mu_{1} \mu_{2}
$$

which is in conjunctive normal form. Following the procedure outlined above, the transformation into a system of linear inequalities results in

$$
\begin{aligned}
& 1-\mu_{1}+q_{1} \leq 1 \\
& 1-\mu_{2}+q_{1} \leq 1
\end{aligned} \Rightarrow \begin{aligned}
& q_{1}-\mu_{1} \leq 0 \\
& q_{1}-\mu_{2} \leq 0
\end{aligned}
$$

which will insure that the original implication is true when the inequalities are enforced on the system behavior using either direct or indirect methods from section 7.3.

\subsection{Constraints Involving Time}

Supervisory controllers are generally driven by sequences of events (firings of transitions) occurring in the plant. Some transitions fire before others, some may fire simultaneously. The states of the plant and controller evolve through time, but there is no direct representation of time. The controller may respond to the firing of transition $t_{i}$ and then respond to the firing of transition $t_{j}$, but there is no indication of how much time has elapsed between the firings of these two transitions. This section will be used to discuss the issues that arise when time is introduced into the control framework.

The most common way of introducing time into a Petri net model of a system is through the use of timed Petri nets $[23,25]$. A timed net works like an ordinary Petri net but includes a new function defined on either the transitions or the places of the net. The function indicates the 
amount of time required for particular transitions to fire or the amount of time that must elapse between the arrival of a token in a place and when it is allowed to take part in enabling and firing another transition. In many cases the function is simply a constant vector that indicates the timing requirements for each of the net's transitions or places, but it may also be quite complicated with the firing times relying on the state of the net, the current time, and other factors.

Timed nets are a useful extension of ordinary nets because they do not alter the basic behavior of these nets, they simply provide more information about their evolution. This means that the standard PN definitions, including structural invariants, still hold true. A controller that enforces certain state constraints and sequential behaviors on an ordinary Petri net will enforce these same behaviors on the net after timing information is added to it.

The invariant based control method is implemented through new places and arcs that connect to existing plant transitions. If the timing information of the plant net were associated with the transitions, then the control method could be used without any changes in the method itself. Because the controller has no new transitions of its own, it is not necessary to establish any new timing properties for the controller. It will react to the firings of the plant and will evolve naturally using the plant's own timing. Control goals such as mutual exclusions, deadlock avoidance, regulation of finite resources, or avoiding forbidden states may be implemented on timed Petri nets using the method exactly as described. When the elapse of time is associated with transition firings, the resulting behavior of the controlled plant may not be entirely what the control designer expected. Consider the simple constraint

$$
\mu_{1}+q_{1} \leq 1
$$

In standard untimed nets, transition firings are considered to be instantaneous. Because of this, the constraint above means two things: 1) place 1 may never have more than 1 token, and 2) transition 1 may not fire if $p_{1}$ contains a token. Now consider if this constraint were to be placed on a Petri net that contained timed transitions. The constraint would take on a third meaning: 3) place 1 may not contain any tokens while transition 1 is in the process of firing. In some cases, this may be exactly what the designer wants, however the designer must be aware of these subtle changes in the meaning of the constraint inequalities when designing the system.

It is possible to maintain the original meaning of the constraint inequalities by using nets that place their timing information on the places rather than the transitions. Transitions undergo instantaneous firing in these nets, just like in ordinary Petri nets. Constraints may be enforced on nets with timed places using the supervisory control techniques of this paper. The controller will add places to the plant net, and because this is a timed net, timing information must be associated with these new controller places. The tokens in a controller place are used to keep track of the plant's state. They are the controller's bookkeeping device, and do not represent a process that requires lengthy amounts of time compared to the time associated with the evolution of the plant. For this reason, the time delay associated with controller places is defined as zero.

Difficulties may arise when supervisory control specifications deal directly with time rather than events. Because the controller has no direct access to a clock, it is not possible to directly realize constraints that reference absolute time or relative timing offsets. Some of these kinds of situations may be tackled by including Petri net structures in the plant that the controller can use 
as an interface between its event-driven world and the world of continuous time. Clock and timer structures are described in the example below.

Example. Consider a plant with five places and seven transitions and the following two constraints.

1. Transition $t_{6}$ may not fire between 6:00 PM and 12:00 AM.

2. Transition $t_{7}$ may not fire until at least 2 minutes has elapsed since the last firing of transition $t_{4}$. If $t_{4}$ has never fired, then the firing of $t_{7}$ is unrestricted.

The supervisory controllers presented here have no access to the current time of day, nor are they informed regarding the amount of time that separates the events to which they react. In order to enforce these constraints, new PN structures will be added to the plant, allowing an interface between the controller and time.

A one-way loop of timed PN places with a single token can be used to create a clock indicating the current time of day. For example, twenty-four places connected in a ring, with a one hour delay for each place, can be used to indicate the current hour of the day. It is not necessary that the delay in every place of the clock be equal, but the firing rules for the clock must indicate that enabled transitions fire instantly. Item 1 above indicates that we are concerned with the six hour period between 6:00 PM and 12:00 AM. The controller will gain access to the current time through the use of the two-place clock shown in Figure 10a. The time delays for the two places are

$$
\text { Times for }\left[\begin{array}{l}
p_{6} \\
p_{7}
\end{array}\right]=\left[\begin{array}{r}
18 \\
6
\end{array}\right] \text { hours }
$$

a)

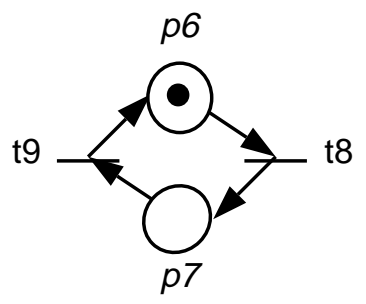

b)

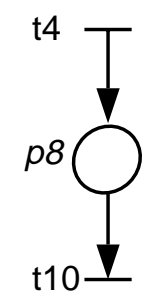

Figure 10: a) A two place time-of-day clock. b) Place 8 contains tokens whenever fewer than two minutes has elapsed since the last firing of $t_{4}$.

The clock is initialized with a single token in $p_{6}$ at 12:00 midnight. After eighteen hours, the token in $p_{6}$ will be available to fire $t_{8}$. The token in $p_{6}$ will transfer to $p_{7}$ at 6:00 PM, and it will remain there until midnight. Thus constraint 1 can be enforced with the inequality $\mu_{7}+q_{6} \leq 1$.

Constraint 2 does not involve the time of day, rather it involves a relative offset in time after the firing of transition 4 . Transition 7 must wait at least two minutes after the last firing of $t_{4}$ before 
it is allowed to fire. A structure is added to the plant to indicate when less than two minutes has elapsed after the last firing of $t_{4}$. This can be done using the net shown in Figure 10b, where the time delay for $p_{8}$ is two minutes. As with the clock of Figure 10, the firing rules must indicate that $t_{10}$ fires whenever it is enabled.

Using the net of Figure 10b, we know that transition 7 should never be allowed to fire when place 8 contains tokens. An initial guess might suggest that we then enforce the constraint $\mu_{8}+q_{7} \leq 1$, but item 2 says nothing about limiting the ability of $t_{4}$ to fire, and this constraint would prohibit $p_{8}$ from ever containing more than one token, indirectly inhibiting the freedom of $t_{4}$. Let $M$ be the maximum number of tokens that could ever appear in place 8. This number could be determined through temporal analysis of the plant, or the designer may simply choose $M$ as a number so ridiculously large that $t_{4}$ could never fire that many times in a reasonable amount of time. Using this value, the following constraint is then placed on the plant,

$$
\mu_{8}+M q_{7} \leq M
$$

This constraint will insure that $t_{7}$ never fires if place 8 contains any tokens. If $M$ is too small, then transition $t_{4}$ may be indirectly inhibited by constraint (77), so it is necessary to determine a large enough value of $M$ to avoid this situation.

In summary, the control designer should be aware of the following points when introducing timing requirements into the invariant based control method.

- Defining time delays on the places of a net, rather than its transitions, avoids certain ambiguities in the meanings of combined state/event constraints. Either method may be used, but the meanings of the constraints in each case must be understood.

- When time is defined on the places of a net, time delays associated with the controls are defined as zero to avoid artificial delays in the evolution of the plant.

- Standard supervisory control constraints dealing with the plant state or mutual exclusions can be implemented seamlessly on place-timed Petri net plants.

- External clock and timer structures with appropriate time delays may be added to the plant to provide an interface between the event sequences of supervisory control and real time.

- The accuracy of clocks and timers is insured by firing rules that insist on the instantaneous firing of enabled transitions for these structures.

- Upper bounds such as $M$ in the example can be used to avoid unwanted consequences of state/event related constraints that deal with the conditions of timers and clocks.

\section{Conclusions}

Petri nets possess many assets as models for discrete event systems. Concurrent processes and events can be easily modeled within the framework. They provide for larger reachable state spaces, 
more compact representation, and increased behavioral complexity compared to automata based models. The goal of this paper has been to present an approach to Petri net supervisory control that is unified and tractable as well as comprehensive and practical.

The primary technical tools required for the use and analysis of the control methods presented here involve Petri net theory and matrix algebra. The main synthesis technique is based on the idea that specifications representing desired plant behavior can be enforced by making them invariants of the closed loop system. Most of the other tools in this paper also revolve around the creation or characterization of invariants or an analysis of the interrelation between control specifications and plant and controller structure.

Because an invariant based controller is itself a Petri net, the unified plant/controller system facilitates the use of established synthesis and analysis methods. The closed loop system can be designed, analyzed, simulated, verified, and augmented using tools already established for Petri nets.

Unobservable transitions have received little attention in the DES PN control literature, but they present an important problem, and systems that incorporate unobservable events are of practical concern. Here the problem of unobservability has been presented and analyzed concurrently and analogously to uncontrollability.

A method has been described for characterizing all feasible invariant based controllers for enforcing a linear constraint on a plant with uncontrollable and unobservable transitions. This characterization can be combined with an extended PN controller definition to enforce the logical union of all these feasible controls. Supervisors designed this way will have a high degree of permissivity. Unfortunately it has not been demonstrated that these controllers will always be maximally permissive, since it is not known if there is a situation where the maximally permissive control law corresponding to a linear predicate is ever something other than a disjunction of other linear predicates.

A wide variety of supervisory control goals can be handled with the proposed method. Inequality (5) is not only appropriate for formulating a large range of forbidden state problems, generalized mutual exclusion constraints [7], and finite resource management problems, but it is also appropriate for specifying a number of supervision goals that are not normally thought of as state-based constraints. Temporary transformations of the graph structure of the plant will allow event-based constraints to be specified in terms of (5); two distinct approaches to enforcing these event-based constraints were presented here. A class of logical predicates on plant behavior can be transformed into linear inequalities for the case of safe nets. The addition of timer and clock structures to a timed Petri net plant model will permit real time based constraints to be represented in the form of (5). A number of approaches to deadlock avoidance and liveness insurance can also be reduced to the enforcement of linear inequalities. The integration of these techniques with the invariant based control method is a current research topic.

Computational efficiency is one of the goals of the supervision techniques presented here. An invariant based controller is computed very efficiently by a single matrix multiplication, and its size grows polynomially with the number of specifications. Since the controller is a Petri net, control actions are also simple to compute online. 
Handling uncontrollable and unobservable transitions does not add any complexity to the online computation of control actions. The increased complexity is encountered only in the initial control design. Computationally tractable techniques have been presented for this process involving the solution of an integer linear program or through the triangularization of integer matrices through constrained row operations.

Invariant based supervisors are viable models for real time control implementations. The speed and efficiency with which they are computed also makes them appropriate for online control reconfiguration due to sensor or actuator faults, or dynamically modified system specifications.

\section{APPENDIX}

\section{A Generating Constraint Transformations}

\section{A.1 An Integer Linear Program for calculating $R_{1}$ and $R_{2}$}

The conditions of Theorem 11 can be converted into an integer linear programming problem (ILP) in the standard form. We will consider only a single constraint on the system; multiple constraints can be handled individually and independently. Thus $n_{c}=1, L$ and $R_{1}$ are vectors, and $b$ and $R_{2}$ are scalars.

Let

$$
R=\left[\begin{array}{lll}
R_{1} & R_{2}^{\prime} & R_{3}
\end{array}\right]
$$

where $R_{2}^{\prime}=R_{2}-1$ and $R_{3} \in \mathbb{Z}^{n_{\mathrm{uc}}}$ is a vector of slack variables. The ILP is defined as

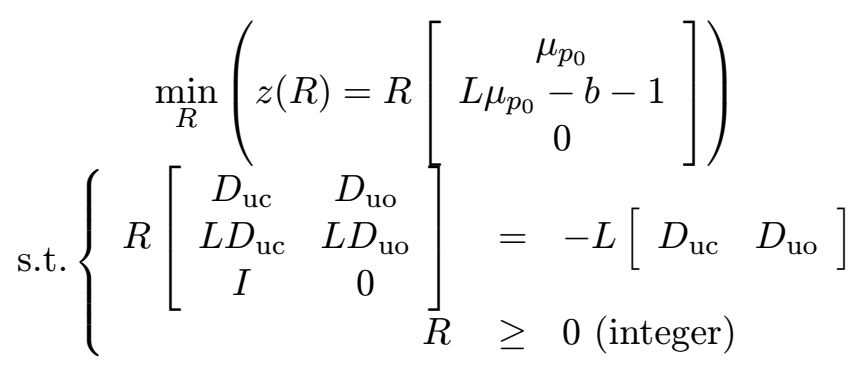

After solving (78), if the minimum of the objective function $z^{*}=z\left(R^{*}\right)$ is greater than $b-L \mu_{p_{0}}$ then the problem can not be solved as there are no values of $R_{1}$ and $R_{2}$ that will satisfy the condition in lemma 10. If the minimum is less than or equal to $b-L \mu_{p_{0}}$, then transform $R_{2}^{\prime}$ back into $R_{2}$ and generate the controller using the formulae in Theorem 11.

Remark. There may be minor difficulties encountered when using this method of generating $R_{1}$ and $R_{2}$. For a controller to exist, we need the objective function of the ILP, $z(R) \leq b-L \mu_{p_{0}}$, however it not clear that we should attempt to minimize this function. Oftentimes, this function will have an unbounded minimum, making it necessary for the designer to introduce an additional constraint in order to achieve a bounded solution. Care must also be taken such that the ILP does not yield the pathological transformation $L^{\prime}=R_{1}+R_{2} L=0$, when there are other nonzero possibilities for $L^{\prime}$. 


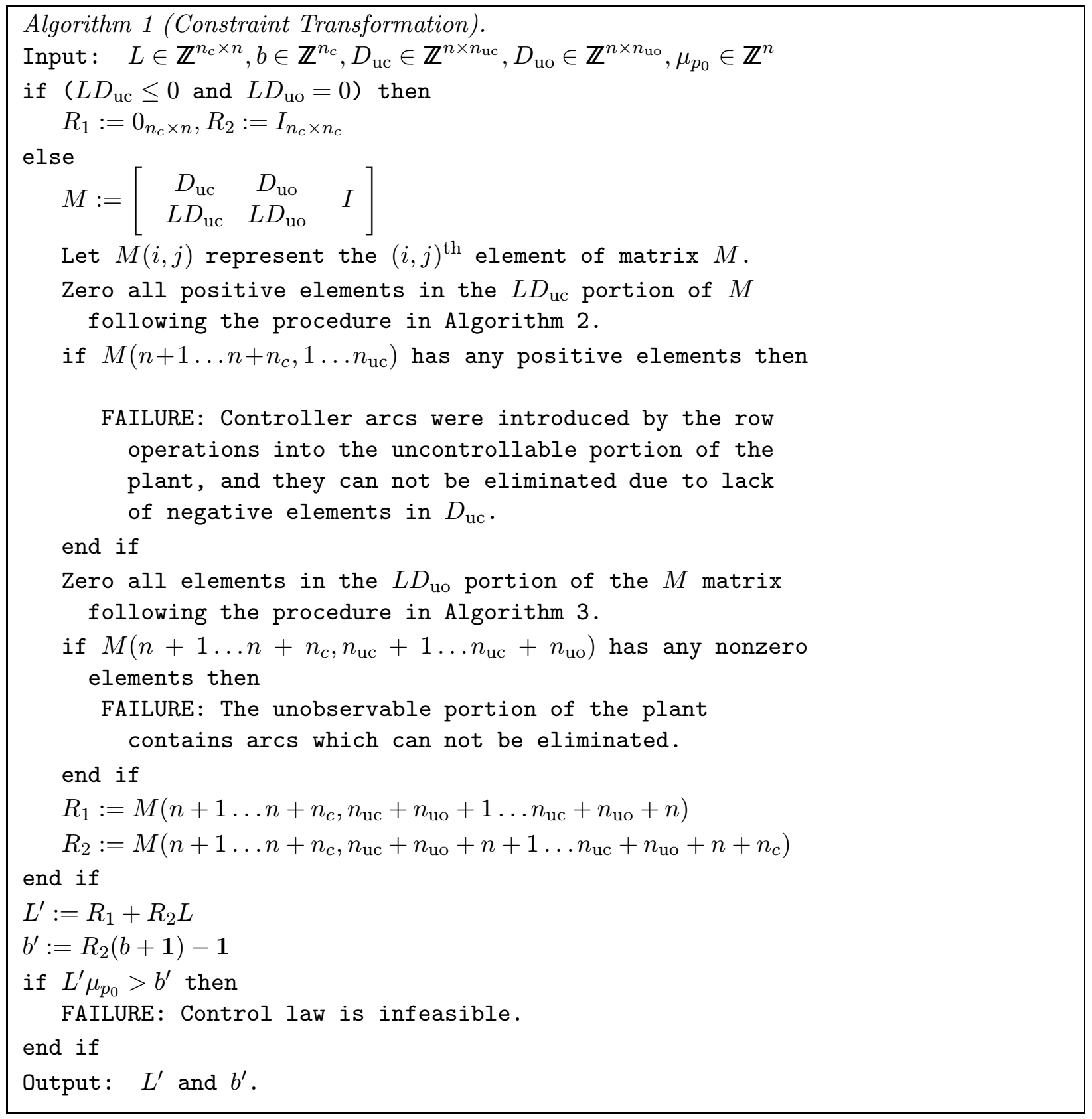

\section{A.2 Matrix Row Operations}

It is possible to obtain appropriate constraint transformations by performing row operations on a matrix containing the uncontrollable and unobservable columns of the plant incidence matrix. The computational part of this procedure involves little more than the integer triangularization of a matrix, and thus it is simpler to compute $R_{1}$ and $R_{2}$ using this method than by using the ILP presented in the previous section. When using the algorithm, recall that $D_{\mathrm{uc}} \in \mathbb{Z}^{n \times n_{\mathrm{uc}}}$ corresponds 
to the uncontrollable transitions in the plant that may be observed, while all unobservable transitions are represented in $D_{\text {uo }} \in \mathbb{Z}^{n \times n_{\text {uо }}}$.

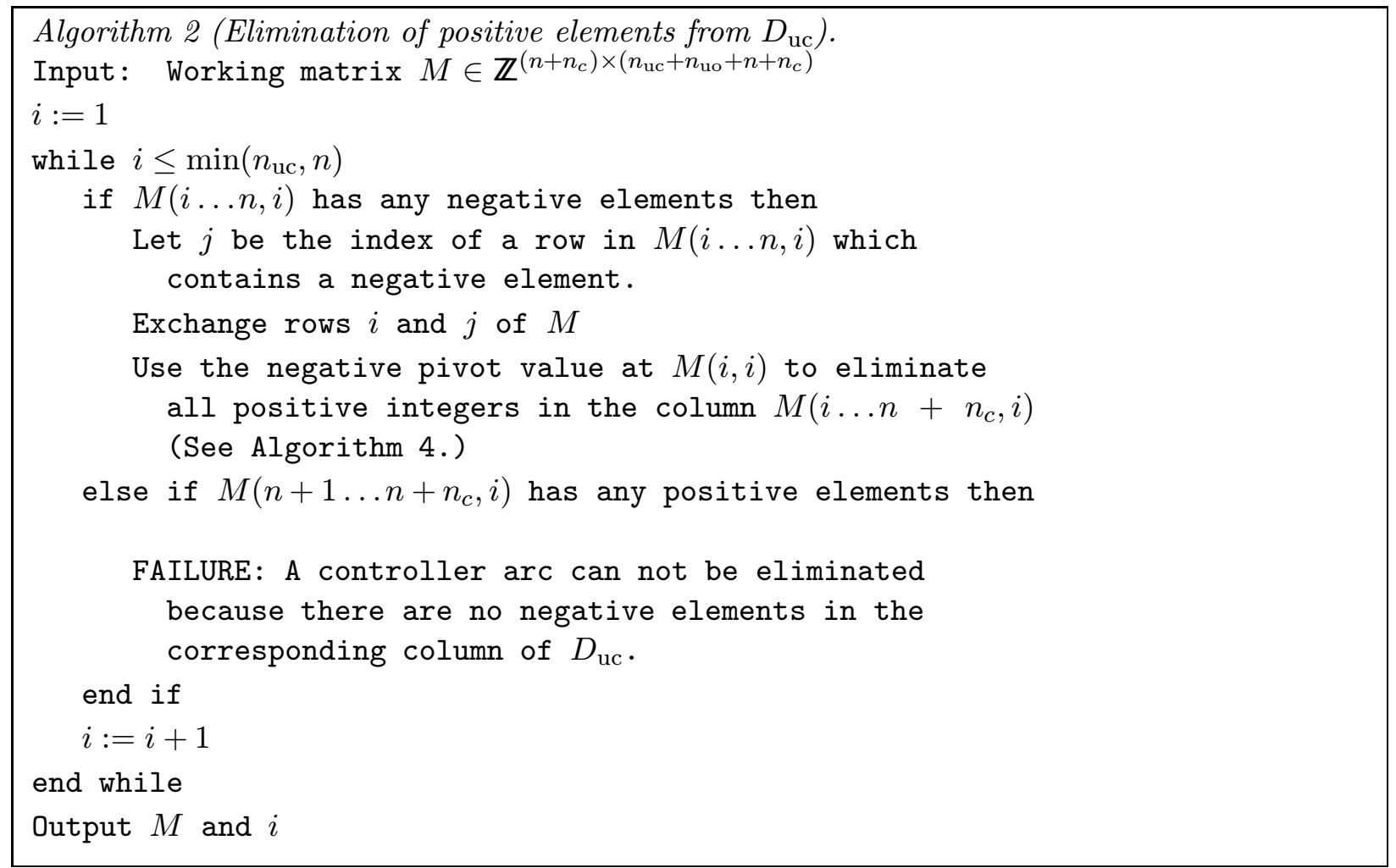

As was done in section A.1, we shall insure that condition (19) is met by making $R_{1} \geq 0$. In terms of row operations, this means that elements in rows are eliminated strictly through addition, never through subtraction, and that rows can be premultiplied only by positive integers. Algorithm 1 presents the procedure for determining $R_{1}$ and $R_{2}$. The procedure for zeroing out the elements in a column of numbers that have the opposite sign of the "pivot" is given in Algorithm 4.

Algorithm 1 insures that condition (20) is met because the procedure for choosing the "pivot" elements never picks from the $L D_{\mathrm{uc}}$ and $L D_{\mathrm{uo}}$ portions of the $M$ matrix. Combined with the zeroing procedure of Algorithm 4, these steps insure that the $R_{2}$ portion of the $M$ matrix is diagonal with strictly positive elements.

Algorithms 2 and 3 (called by Algorithm 1) are used to make sure that the transformed constraints meet conditions (17) and (18). The feasibility check at the end of Algorithm 1 directly tests the condition of lemma 10 to insure that the controller does exist. The instructions for picking positive or negative elements to act as pivots in the two main loops are left specifically vague. Different methods of choosing the pivot will lead to different constraint transformations. It would be possible, for instance, to find a basis for all valid constraint transformations by repeating the procedures in Algorithm 1 whenever there was a choice of more than one pivot for a given column. 


\section{References}

[1] K. Barkaoui and I. B. Abdallah, "Deadlock avoidance in FMS based on structural theory of petri nets", In IEEE Symposium on Emerging Technologies and Factory Automation, volume 2, pp. 499-510, Piscataway, NJ, 1995. IEEE.

[2] S. Bogdan and F. L. Lewis, "Matrix approach to deadlock avoidance of dispatching in multiclass finite buffer reentrant flow lines", In Proceedings of the 1997 IEEE International Symposium on Intelligent Control, pp. 397-402, Piscataway, NJ, July 1997. IEEE.

[3] J. Desel and J. Esparza, Free Choice Petri Nets, Cambridge University Press, 1995.

[4] A. A. Desrochers and R. Y. Al-Jaar, Applications of Petri Nets in Manufacturing Systems, IEEE Press, Piscataway, NJ, 1995.

[5] J. Ezpeleta, J. M. Colom, and J. Martínez, "A Petri net based deadlock prevention policy for flexible manufacturing systems", IEEE Transactions on Robotics and Automation, vol. 11, no. 2, pp. 173-184, April 1995.

[6] S.-C. Fang and S. Puthenpura, Linear Optimization and Extensions: Theory and Algorithms, Prentice Hall, Engelwood Cliffs, NJ, 1993.

[7] A. Giua, F. DiCesare, and M. Silva, "Generalized mutual exclusion constraints on nets with uncontrollable transitions", In Proceedings of the 1992 IEEE International Conference on Systems, Man, and Cybernetics, pp. 974-979, Chicago, IL, October 1992.

[8] L. E. Holloway, B. H. Krogh, and A. Giua, "A survey of Petri net methods for controlled discrete event systems", Discrete Event Dynamic Systems: Theory and Applications, vol. 7, no. 2, pp. 151-190, April 1997.

[9] J. N. Hooker, "A qualitative approach to logical inference", Decision Support Systems, no. 4, pp. 45-69, 1988.

[10] H.-H. Huang, F. L. Lewis, and D. A. Tacconi, "Deadlock analysis using a new matrix-based controller for reentrant flow line design", In IECON Proceedings (Industrial Electronics Conference), volume 1, pp. 463-468. IEEE, Los Alamitos, CA, 1996.

[11] F. L. Lewis, H. Huang, D. Tacconi, A. Gürel, and O. Pastravanu, "Analysis of deadlocks and circular waits using a matrix model for discrete event systems", Technical report, Automation and Robotics Research Institute, The University of Texas at Arlington, Ft. Worth, TX, October 1995.

[12] Y. Li and W. M. Wonham, "Controllability and observability in the state-feedback control of discrete-event systems", In Proceedings of the 27th Conference on Decision and Control, pp. 203-208, Austin, TX, December 1988.

[13] Y. Li and W. M. Wonham, "Control of vector discrete event systems II - controller synthesis", IEEE Transactions on Automatic Control, vol. 39, no. 3, pp. 512-530, March 1994. 
[14] J. Martinez and M. Silva, "A simple and fast algorithm to obtain all invariants of a generalized Petri net", In Advances in Petri Nets, number 52 in Lecture Notes in Computer Science. Springer-Verlag, Berlin, 1980.

[15] J. O. Moody, Petri Net Supervisors for Discrete Event Systems, PhD thesis, Department of Electrical Engineering, University of Notre Dame, Notre Dame, IN., 1998.

[16] J. O. Moody and P. J. Antsaklis, Supervisory Control of Discrete Event Systems Using Petri Nets, Kluwer Academic Publishers, 1998.

[17] J. O. Moody, P. J. Antsaklis, and M. D. Lemmon, "Automated design of a Petri net feedback controller for a robotic assembly cell", In Proceedings of 1995 INRIA/IEEE Symposium on Emerging Technologies and Factory Automation, volume 2, pp. 117-128, Paris, France, October 1995.

[18] J. O. Moody, P. J. Antsaklis, and M. D. Lemmon, "Feedback Petri net control design in the presence of uncontrollable transitions", In Proceedings of the 34th IEEE Conference on Decision and Control, volume 1, pp. 905-906, New Orleans, LA, December 1995.

[19] J. O. Moody, K. Yamalidou, M. D. Lemmon, and P. J. Antsaklis, "Feedback control of Petri nets based on place invariants", In Proceedings of the 33rd IEEE Conference on Decision and Control, volume 3, pp. 3104-3109, Lake Buena Vista, FL, December 1994.

[20] T. Murata, "Petri nets: Properties, analysis, and applications", Proceedings of the IEEE, vol. 77 , no. 4 , pp. 541-580, 1989.

[21] J. L. Peterson, Petri Net Theory and the Modeling of Systems, Prentice Hall, Engelwood Cliffs, NJ, 1981.

[22] P. J. G. Ramadge and W. M. Wonham, "The control of discrete event systems", Proceedings of the IEEE, vol. 77, no. 1, pp. 81-97, 1989.

[23] C. V. Ramamoorthy and G. S. Ho, "Performance evaluation of asynchronous concurrent systems using Petri nets", IEEE Transactions on Software Engineering, vol. 6, no. 5, 1980.

[24] W. Reisig, Petri Nets, Springer-Verlag, Berlin; New York, 1985.

[25] J. Sifakis, "Performance evaluation of systems using Petri nets", In Advances in Petri Nets, W. Brauer, Eds., number 84 in Lecture Notes in Computer Science. Springer-Verlag, Berlin; New York, 1979.

[26] R. S. Sreenivas, "On commoner's liveness theorem and supervisory policies that enforce liveness in free-choice Petri nets", Systems \& Control Letters, vol. 31, no. 1, pp. 41-48, June 1997.

[27] R. S. Sreenivas, "On the existence of supervisory policies that enforce liveness in discrete-event dynanic systems modeled by controlled Petri nets", IEEE Transactions on Automatic Control, vol. 42, no. 7, pp. 928-945, July 1997.

[28] W. M. Wonham and P. J. G. Ramadge, "On the supremal controllable sublanguage of a given language", SIAM Journal of Control Optimization, vol. 25, no. 3, pp. 637-659, 1987. 
[29] K.-Y. Xing, B.-S. Hu, and H.-X. Chen, "Deadlock avoidance policy for Petri net modeling of flexible manufacturing systems with shared resources", IEEE Transactions on Automatic Control, vol. 41, no. 2, pp. 289-295, February 1996.

[30] E. Yamalidou, Modeling, Optimization and Control of Discrete-Event Chemical Processes using Petri Net Theory, PhD thesis, Department of Chemical Engineering, University of Notre Dame, Notre Dame, IN., February 1991.

[31] K. Yamalidou and J. C. Kantor, "Modeling and optimal control of discrete-event chemical processes using Petri nets", Computers in Chemical Engineering, vol. 15, no. 7, pp. 503-519, 1991.

[32] K. Yamalidou, J. O. Moody, M. D. Lemmon, and P. J. Antsaklis, "Feedback control of Petri nets based on place invariants", Automatica, vol. 32, no. 1, pp. 15-28, January 1996. 


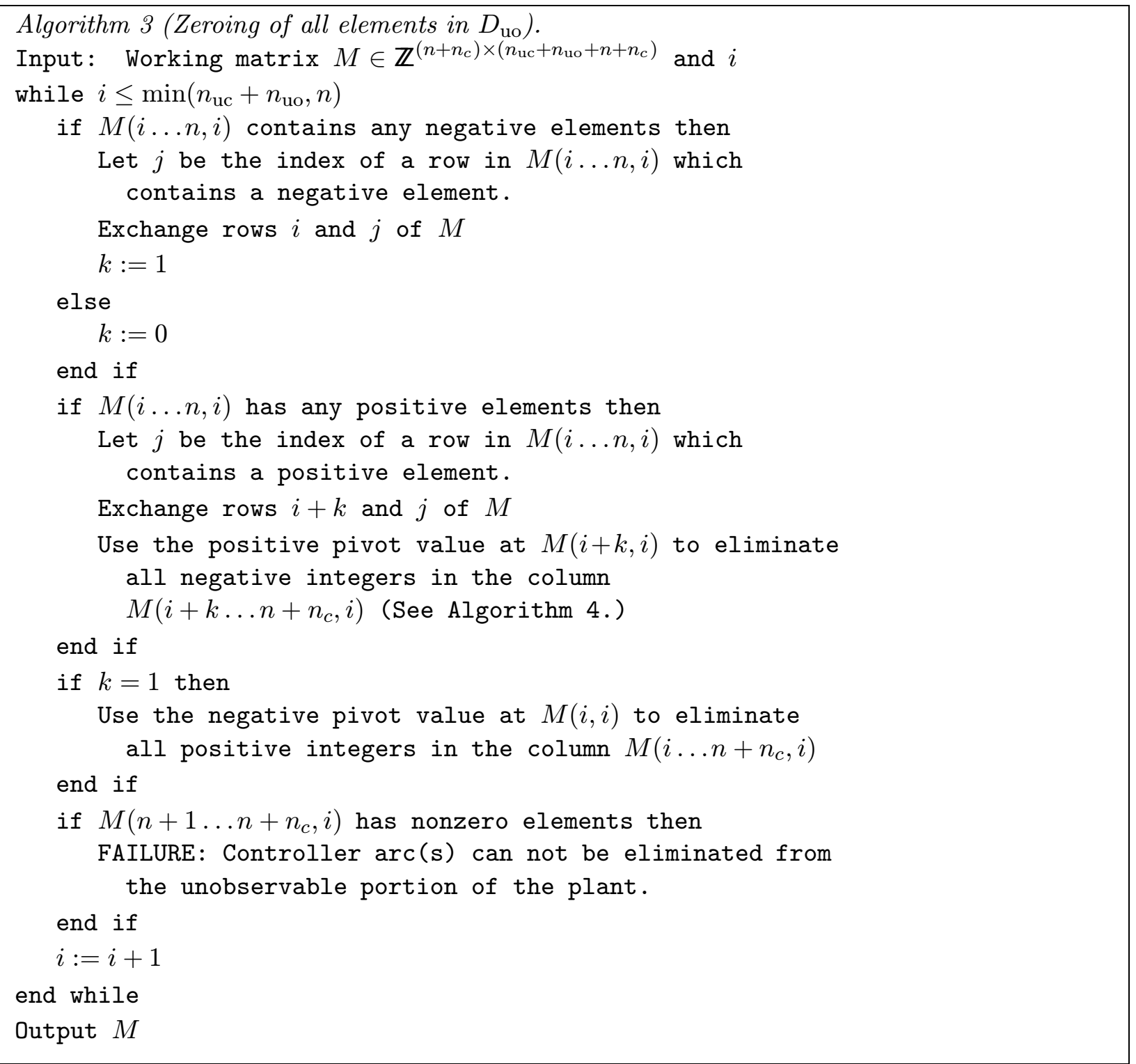




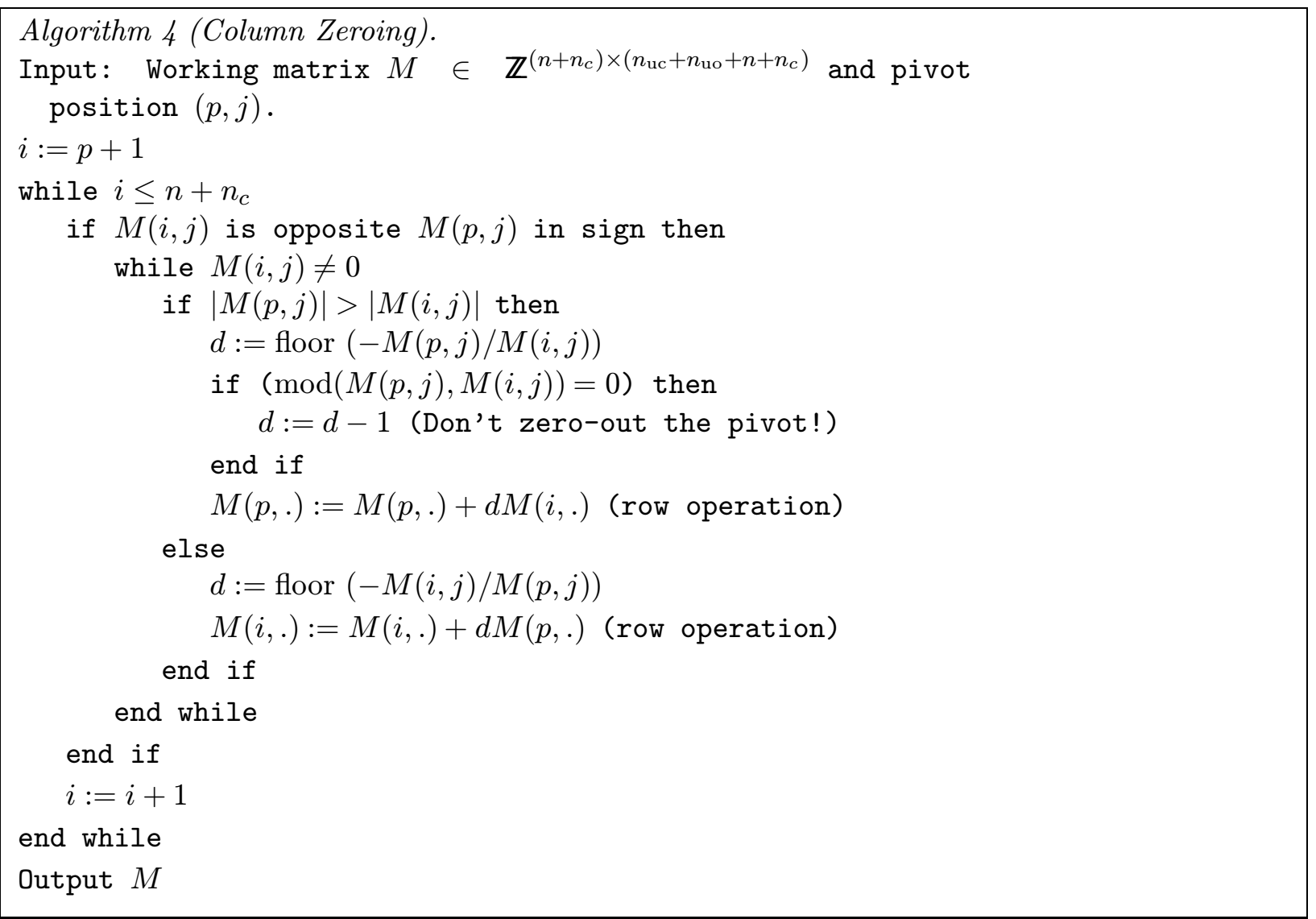

